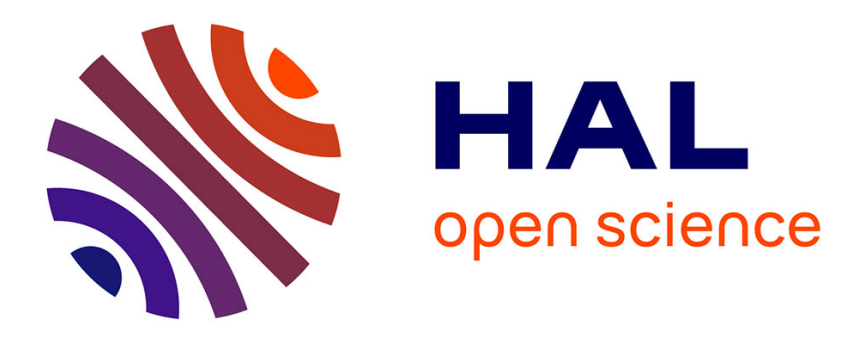

\title{
Droit des actionnaires et concentration de la propriété en Europe
}

Tristan Auvray

\section{To cite this version:}

Tristan Auvray. Droit des actionnaires et concentration de la propriété en Europe. Revue Economique, 2014, 65 (1), pp.159-199. 10.3917/reco.651.0159 . hal-00843004

\section{HAL Id: hal-00843004 \\ https://hal.science/hal-00843004}

Submitted on 18 Feb 2020

HAL is a multi-disciplinary open access archive for the deposit and dissemination of scientific research documents, whether they are published or not. The documents may come from teaching and research institutions in France or abroad, or from public or private research centers.
L'archive ouverte pluridisciplinaire HAL, est destinée au dépôt et à la diffusion de documents scientifiques de niveau recherche, publiés ou non, émanant des établissements d'enseignement et de recherche français ou étrangers, des laboratoires publics ou privés. 


\title{
Droit des actionnaires et concentration de la propriété en Europe
}

\author{
Tristan Auvray*
}

Version auteur d'un article publié dans la Revue économique et disponible en ligne : https://www.cairn.info/revue-economique-2014-1-page-159.htm

Citation :

Auvray T., (2014) "Droit des actionnaires et concentration de la propriété en Europe”, Revue économique, 65(1), p. 159-199. DOI: 10.3917/reco.651.0159

Cet article propose des indicateurs juridiques portant sur le pouvoir décisionnaire des actionnaires en assemblée générale dans 15 pays européens. Elaborés à l'aide d'une étude de droit comparé (Shearman \& Sterling LLP [2007]), ces indicateurs reflètent la capacité des actionnaires à nommer et révoquer les administrateurs des sociétés cotées d'une part ( droits de contrôle»), ainsi que leur implication dans un ensemble de décisions en assemblée générale d'autre part («droits de structure»). Les indicateurs classiques de la Law and Finance et les mesures élaborées ici sont ensuite confrontées pour expliquer la concentration actionnariale de 287 sociétés cotées. Cette étude montre l'existence d'une complémentarité institutionnelle entre la concentration de la propriété des actions, la régulation des conflits d'agence et le pouvoir des actionnaires en assemblée générale.

\section{SHAREHOLDER RIGHTS AND OWNERSHIP CONCENTRATION IN EUROPE}

This paper presents legal indicators dealing with shareholder rights in general meeting. Assembled with the help of a comparative legal study (Shearman \& Sterling LLP [2007]), the indicator is calculated for 15 European countries. These measures reflect the ability of shareholders to appoint and dismiss directors of listed companies ("control rights") and the requirement of their vote on multiple decisions in general meeting ("structure rights"). A study on a sample of 287 European companies shows that these new indicators predict corporate control outcomes: the higher the control and structure rights are the more corporations are controlled by shareholders rather than by directors. This study highlights the institutional complementarities between the indicators we build, the Law and Finance indicators of legal protection of minority shareholders and ownership concentration.

Classification JEL : G2, G34, K22

\footnotetext{
* Université Paris 13 (Sorbonne, Paris, Cité), CEPN-CNRS, 99 bd. J-B Clément, 93430 Villetaneuse, France. Courriel : tristan.auvray@univ-paris13.fr

Je remercie Caroline Granier pour son aide dans la collecte des données. Je remercie également Olivier Brossard, Matthieu Montalban, François Morin ainsi que les rapporteurs anonymes pour leurs commentaires qui ont grandement contribué à l'amélioration de ce texte.
} 


\section{INTRODUCTION}

La mesure des institutions juridico-économiques constitue le cœur des travaux appliqués de la Law and Finance. Les principaux auteurs de ce courant de recherche que sont La Porta, Lopez-de-Silanes, Shleifer (LLS) et Vishny (LLSV) présentent dans des articles de 1997 et 1998 un "indicateur anti-administrateur " qui, avec leurs autres contributions, suscite rapidement l'engouement et la critique du monde académique ${ }^{1}$. En réponse à certaines de ces réactions, Djankov et LLS (DLLS [2008]) amendent cette mesure de la protection légale des actionnaires minoritaires tout en élaborant un nouvel « indicateur anti-malversation » qui lui est complémentaire. Ces deux métriques juridiques portent sur les deux problèmes agentiels opposant respectivement les actionnaires minoritaires aux administrateurs d'une part et aux actionnaires de contrôle d'autre part. Ces mesures ont alors pour objectif de différencier les pays en fonction du risque d'expropriation des minoritaires par les titulaires du contrôle des sociétés cotées.

Revenir aux origines de ces travaux permet de reposer le problème fondamental abordé par ces auteurs mais qu'ils délaissent au profit d'une analyse juridique des conflits d'agence qui structure alors le champ de la Law and Finance. LLSV [1998, p. 1114] soulignent en effet que la caractéristique distinctive des actions réside dans les droits de vote qui y sont attachés. Ils en expliquent toute l'importance, à la suite de Hart [1995], en ce sens qu'ils permettent de nommer ou révoquer les administrateurs des sociétés de capitaux. Toutefois, après avoir placé au point de départ de leur analyse ce processus que Berle et Means [1932] dénomment « le contrôle ", ils en repoussent l'examen des propriétés juridiques pour lui privilégier in fine une analyse de la protection légale des actionnaires minoritaires.

L'objet de cet article est de renouer avec une approche juridico-économique du contrôle. Il s’agit ici de développer des indicateurs du pouvoir actionnarial se situant en amont des conflits d'agence et des dispositifs juridiques visant à les résoudre. Le pouvoir juridique actionnarial ne se limite pas en effet à la protection dont jouissent les minoritaires mais tient aussi à la place qui est faite aux actionnaires lors du choix des administrateurs et lors des décisions les plus stratégiques. Une étude de droit comparé portant sur une quinzaine de pays européens (Shearman \& Sterling LLP [2007]) nous permet de construire des indicateurs allant dans ce sens. Elle autorise principalement l'élaboration d'une mesure des «droits de contrôle » visant à capturer certaines caractéristiques juridiques de la nomination et de la révocation des administrateurs. Cette étude permet également la construction d'un indicateur des "droits de structure » portant sur un ensemble de décisions affectant l'actif, le passif et les statuts de la société de capitaux. Ces deux mesures sont synthétisables en un indicateur du " pouvoir actionnarial » illustrant l'intensité, requise par chaque juridiction, de l'implication des propriétaires de titres dans les organes délibérants des sociétés cotées.

L'enjeu de ces divers indicateurs est d'expliquer la diversité, au travers des pays, de la concentration de l'actionnariat des firmes, ce qui éclaire la manière dont elles sont gouvernées $^{2}$. Sont-elles sous contrôle actionnarial ou sous la coupe de leurs managers ou administrateurs ? Selon les principaux auteurs de la Law and Finance (e.g. LLSV [1998] et DLLS [2008]), la concentration du capital des sociétés cotées s'explique par le manque de régulation des conflits d'agence et, notamment, par l'incapacité juridique des actionnaires minoritaires à se protéger des titulaires du contrôle.

\footnotetext{
${ }^{1}$ Cf. par exemple Vilanova [2007] et Spamann [2010] pour une revue critique de ces travaux.

${ }^{2}$ La finalité de LLSV [1998] et DLLS [2008] est d'expliquer les différences de développement des marchés de la finance externe - assimilable selon eux à la dispersion des actions. D’autres travaux (e.g. Holderness [2008]), y compris le présent article, considèrent la question de la propriété des actions pour elle-même.
} 
Cet article s'attache à défendre l'idée que la protection des minoritaires vis-à-vis des administrateurs ou des grands actionnaires ne saurait déterminer à elle seule la concentration de la propriété des actions. Celle-ci s'explique tant par l'importance que le droit concède à la décision actionnariale que par la régulation des problèmes engendrés par la délégation de cette décision. Cette proposition est validée par une analyse empirique portant sur 287 sociétés cotées dans 15 pays européens, une fois prises en compte les caractéristiques individuelles des firmes. Il est notamment démontré que la combinaison des deux types d'indicateurs juridiques est davantage explicative que chacune de ces mesures prises isolément.

Dans le courant Law and Finance, deux cadres théoriques sont alors en concurrence pour établir et interpréter la réalité de cette cohérence entre l'environnement juridique des sociétés cotées et la concentration de leur actionnariat. Selon la théorie de l'origine légale (LLS [2008]) la substance des règles de droit serait structurellement déterminée par la tradition juridique des pays qui influencerait donc la gouvernance des firmes ${ }^{3}$. Pour d'autres auteurs, au contraire, les règles de droit seraient davantage produites selon un "canal de l'économie politique » (Roe et Siegel [2009] ; Roe [2006, 2003]) ou un « canal institutionnel » (Armour et al. [2009a, b]). Le cadre d'analyse de cet article s'inscrit dans cette seconde approche qui montre que l'état de la régulation et de la concentration du capital s'expliquent moins par l'origine juridique des pays que par des causes macroéconomiques (Pollin et Vaubourg [2006]), microéconomiques (Vilanova [2007] ; Holderness [2008, 2009]) ou socio-politiques (Roe [2003, 2006] ; Roe et Siegel [2009] ; Fligstein et Choo [2005] ; Gourevitch et Shinn [2005]; Armour et al. [2009a, b]). Les règles au niveau national sont influencées par le contexte économique dans lequel elles sont élaborées, et réciproquement. Ici, le mode de contrôle des sociétés cotées, à dominante actionnarial ou « administratorial », et les dispositifs juridiques, tels que les mesurent les divers indicateurs, sont donc interprétés selon leur complémentarité institutionnelle, sans chercher à expliquer si l'un est le déterminant de l'autre ${ }^{4}$. La notion de complémentarité institutionnelle d'Aoki [2001] est en effet au cœur de cette seconde approche (e.g. Armour et al. [2009a, b]). Aussi, la méthodologie empirique de cet article s'inspire de cette notion telle qu'elle peut être mobilisée dans une économie politique du droit et de la finance que l'on trouve, par exemple, dans les travaux sur la diversité des capitalismes (Amable [2005] ; Amable et Lung [2008]) ou sur leur convergence formelle et fonctionnelle (Armour et al. [2009b] ; Deakin et Rebérioux [2009]).

Au final, notre contribution est triple : i) la construction de nouveaux indicateurs souligne la nécessité de comprendre le droit des actionnaires comme un droit d'accès à la gouvernance, à la représentation au conseil d'administration ; ii) une étude empirique faisant émerger trois groupes de pays européens démontre la complémentarité entre les environnements juridiques et la concentration de la propriété des actions ; iii) ce résultat est interprété selon les termes d'une complémentarité institutionnelle, ce qui permet notamment d'expliquer pourquoi le développement de la protection des actionnaires minoritaires ne conduit pas forcément à un processus de dispersion de l'actionnariat ; il convient en effet de tenir compte de l'existence du pouvoir juridique des titulaires du contrôle.

\footnotetext{
${ }^{3}$ La théorie de l'origine légale compare l'efficacité des systèmes juridiques de common law et de droit civiliste et soutient de manière constante la thèse de la supériorité économique des premiers sur les seconds, bien que ses auteurs se défendent d'une telle conception (LLS [2008]). Cette efficacité tiendrait notamment à la méthode de résolution des conflits lors des défaillances de marché. La tradition juridique britannique encouragerait systématiquement une solution d'ordre privée, en réduisant le coût de celle-ci (LLS [2008]), et serait marquée par un formalisme judiciaire efficace car peu coûteux en temps et en argent (DLLS [2003]). Pour une lecture critique de cette thèse on pourra consulter par exemple Deffains [2007].

${ }^{4}$ Aussi, le terme « explicatif », lorsqu'il est employé pour mettre en évidence la relation entre les indicateurs juridiques et la concentration de l'actionnariat, ne doit être compris que dans le sens d'une corrélation statistique.
} 
La prochaine partie présente brièvement le contenu des principaux indicateurs juridiques développés par les diverses approches du courant Law and Finance. Elle se poursuit avec la présentation du processus de codage des mesures élaborées ici. La seconde partie développe l'étude empirique testant l'existence d'une complémentarité institutionnelle entre la concentration actionnariale et l'environnement juridique des sociétés cotées tel que le captent les divers indicateurs. La dernière partie interprète les trois configurations économiques et institutionnelles issues de cette analyse. La conclusion rappelle les trois apports de cette étude.

\section{LES MESURES DU DROIT DANS LA GOUVERNANCE D’ENTREPRISE}

Cette partie présente succinctement les divers indicateurs portant sur le droit des actionnaires dans une approche statique et comparative ou longitudinale et fonctionnelle. De nouveaux indicateurs juridiques sont alors proposés et leurs relations avec les autres mesures sont précisées.

\section{Les indicateurs juridiques de la protection des actionnaires minoritaires}

Les principaux auteurs de la Law and Finance ont construit trois types de mesures portant sur la protection des actionnaires minoritaires (DLLS [2008] ; LLS [2006] ; LLSV [1998]). LLS [2006] élaborent des indicateurs sur la base du droit boursier. Ceux-ci comprennent, notamment, un indicateur informationnel sur l'étendue de la divulgation du prospectus d'offre publique initiale et un indicateur sur la difficulté procédurale de recouvrir des pertes liées à de mauvaises déclarations du prospectus. La description suivante se focalise sur les indicateurs anti-administrateur (LLSV [1998] ; DLLS [2008]) et anti-malversation (DLLS [2008]) dans la mesure où ils intègrent certains aspects juridiques relatifs à l'intervention des actionnaires minoritaires en assemblée générale.

Les critiques généralement avancées à l'égard de ce type d'indicateurs ne sont pas développées ici (sur ce sujet cf. Pagano et Volpin [2005] ; Spamann [2010] ; Lele et Siems [2007]; Siems [2008]; Vilanova [2007]). On notera seulement que l'indicateur antiadministrateur a concentré sur lui l'ensemble des remarques méthodologiques et que l'indicateur anti-malversation évite plusieurs de ces écueils. Certaines de ces critiques ont d'ailleurs conduit à la révision de l'indicateur anti-administrateur (DLLS [2008]). Quoi qu'il en soit, ces deux mesures sont retenues ici car elles portent sur une problématique particulière, celle des conflits d'agence, et qu'elles véhiculent malgré tout une certaine information sur le droit positif en vigueur dans plusieurs pays en 2003.

L'indicateur des droits anti-administrateur de LLSV [1998] se focalise sur un problème d'agence classique portant sur l'opportunisme, l'enracinement, ou l'incompétence managériale. Il permet essentiellement de rendre compte de la capacité des actionnaires minoritaires à intervenir en AG. LLSV [1998] et LLS [1999] ont toutefois mis en avant que, dans la plupart des sociétés cotées à travers le monde, les structures de propriété sont concentrées. Ils se focalisent alors sur un nouveau problème agentiel qu'est le risque d'expropriation des actionnaires minoritaires, c'est-à-dire le risque d'un transfert de ressources d'une société cotée à son actionnaire de contrôle via une autre société que celui-ci détient intégralement. L’expression «anti-self-dealing» est donc ici traduite par «anti- 
malversation $»^{5}$. L'indicateur des droits anti-malversation de DLLS [2008] vise alors à capturer les aspects juridiques de ce problème et de sa résolution. Cette mesure, davantage concernée par la procédure judiciaire elle-même, prend néanmoins en compte les possibilités d’implication des actionnaires minoritaires en assemblée générale.

Dans les deux cas, ces indicateurs ont pour objectif de saisir le degré de protection des actionnaires minoritaires vis-à-vis des administrateurs, que ceux-ci soient des managers, des représentants des blocs de contrôle ou bien des administrateurs indépendants au regard de ces deux catégories. Cette protection a pour finalité de prévenir une action des membres des conseils d'administration qui viendrait diminuer la valeur des titres des actionnaires minoritaires.

En dépit de l'intérêt que LLS [1999] portent à la compréhension du pouvoir de contrôle de certains actionnaires, l'analyse des auteurs de la théorie de l'origine légale n'aura pas porté sur les dispositifs juridiques qui permettent d'asseoir ce contrôle. Certains indicateurs longitudinaux comblent cette lacune de manière partielle.

\section{Les indicateurs longitudinaux}

Face à l'approche comparative et statique de LLSV [1998] et DLLS [2008] deux groupes de chercheurs ont simultanément développé des indicateurs dans une perspective longitudinale et fonctionnelle ${ }^{6}$. Il s'agit du «shareholder protection index » du Centre for Business Research de l’Université de Cambridge (Lele et Siems [2007] ; Siems [2008]) et des "shareholder rights protection index" et "minority shareholders protection index" développés par des chercheurs de l’Université de Tilburg (Martynova et Renneboog [2008, 2011]). Ces mesures juridiques s'inscrivent dans une problématique de la convergence formelle et fonctionnelle du droit relatif aux actionnaires des sociétés cotées sur la période allant des années 1970 ou 1990 jusqu'en 2005. Elles embrassent de larges pans du droit des actionnaires mais demeurent incomplètes sur les processus de nomination et de révocation des administrateurs (eg. Martynova et Renneboog [2008, 2011] ; Siems [2008]) ou portent sur un périmètre géographique restreint (eg. Lele et Siems [2007]).

Certains des éléments juridiques considérés ici se retrouvent donc dans ces mesures. Ainsi, la procédure de révocation ad nutum est prise en compte dans le "shareholder protection index " mais la procédure de nomination en est absente (Siems [2008]). Le périmètre géographique de cet indicateur, essentiellement mondial, ne permet pas d'établir une comparaison complète entre ses composantes et celles qui leur sont proches dans l'indicateur du pouvoir actionnarial présenté ci-dessous, davantage focalisé sur les pays européens. Dans la mesure du possible, les corrélations seront néanmoins indiquées pour les éléments et pays communs aux deux indicateurs.

La comparaison avec les constituants du «shareholder rights protection index » est tout simplement impossible puisque ses auteurs n'en publient qu'un détail partiel. Ce faisant, ils ne

\footnotetext{
${ }^{5}$ Une traduction plus littérale de « self-dealing transactions » serait celle de « transactions avec apparentés ».

${ }^{6}$ D'autres travaux présentent une analyse longitudinale du droit des actionnaires. Les rapports annuels Doing Business de 2006 à 2012, publiés par la Banque mondiale, offrent une dynamique de l'indicateur antimalversation de DLLS [2008]. Pagano et Volpin [2005] calculent l'indicateur anti-administrateur de LLSV [1998] de 1993 à 2001. Des indicateurs alternatifs et dynamiques sont par ailleurs développés par Pistor et al. [2000] pour 24 économies en transition, par Hyytinen et al. [2003] pour la Finlande et par Blazy et al. [2012] pour la France. Ces derniers indicateurs plus sophistiqués prennent également en compte la substituabilité fonctionnelle des différentes facettes du droit des actionnaires.
} 
rendent pas leurs mesures réfutables, ce qui va à l'encontre de la méthodologie conventionnelle en la matière (Spamann [2010] ; Lele et Siems [2007] ; Siems [2008]). On notera néanmoins que le "shareholder rights protection index » de Martynova et Renneboog [2008, 2011] comprend, entre autres, un indicateur des droits de nomination et de révocation («the appointment rights index») allant de 0 à 12 . Ce score comprend cinq variables ${ }^{7}$ dont l'une, à deux modalités, porte sur la nomination du conseil par les actionnaires. Nous proposons ici au contraire un indicateur plus complet sur le seul processus de nomination et de révocation des administrateurs comprenant six variables et 16 modalités. Il n'est toutefois pas possible de le comparer à la variable de Martynova et Renneboog [2008, 2011] car les valeurs associées ne sont pas reportées dans leurs articles. Par ailleurs, ces auteurs ne définissent la variable que par son label sans détailler son contenu précis, ce qui rend sa reproduction impossible.

Enfin, à la lumière de ces études longitudinales, il convient de préciser que les indicateurs élaborés ici traduisent l'état du droit à la suite du processus de convergence que décrivent ces travaux. Leurs conclusions seront donc mobilisées dans l'interprétation des résultats de l'étude statique et comparative menée ci-après.

\section{De nouveaux indicateurs juridiques du pouvoir actionnarial}

Les indicateurs construits ici reposent sur une étude de droit comparé commandée par la Commission Européenne et traitant du principe de proportionnalité dit "une action, une voix » (Shearman \& Sterling LLP [2007]) ${ }^{8}$. L’indicateur du « pouvoir actionnarial » proposé repose essentiellement sur la construction d'une mesure des «droits de contrôle ». L'objectif est de capturer certains éléments du droit positif en vigueur en 2006 et portant sur la nomination et la révocation des membres d'un conseil d'administration ou de surveillance. Ce processus, jusque là rarement pris en compte, est pourtant au cœur des grandes analyses sur l'actionnariat (Berle et Means [1932] ; Chevalier [1970]; Morin [1974]; LLS [1999]; Claessens et al. [2000] ; Faccio et Lang [2002]). Il convient donc de le considérer dans une étude juridico-économique visant à décrire les niveaux de concentration de la propriété des actions des sociétés cotées. Les droits de contrôle sont complétés par une mesure des « droits de structure » afin de saisir plus largement le pouvoir que le droit concède aux actionnaires en assemblée générale. Cet indicateur agrège un ensemble de décisions hétéroclites portant par exemple sur la structure du capital et des actifs de la société, sur sa nationalité ou son objet social.

Une clarification est ici nécessaire. Pour des raisons pratiques, la définition du contrôle adoptée ici se restreint volontairement au pouvoir de nomination et de révocation des administrateurs par ceux qui parviennent à rassembler une majorité en AG. Pour les besoins de l'analyse, on adopte en ce sens la définition du contrôle qu'en donnent Chevalier [1970] et

\footnotetext{
${ }^{7}$ Ces variables portent sur (i) « la représentation des employés » dans le conseil d’administration (0/2); (ii) « la nomination du conseil par les actionnaires » (0/2) ; (iii) « la durée des mandats des administrateurs » $(0 / 1 / 2)$; (iv) la régulation des participations réciproques $(0 / 1)$ et circulaires $(0 / 1) ;(v)$ la régulation de la procédure de vote : « vote par procuration et par courrier » $(0 / 2)$ et « dépôt/enregistrement des actions avant une assemblée générale » (dépôt et/ou enregistrement des actions au porteur (0/1/2), dépôt des actions nominatives (0/2)).

${ }^{8}$ Cette étude constitue une partie du rapport "Proportionality between ownership and control in EU listed companies ", préparé pour la Commission Européenne par l'Institutional Shareholder Services (ISS), le European Corporate Governance Institute (ECGI) et le cabinet d'avocats Shearman \& Sterling LLP, dans le cadre de l'appel d’offres «Proportionnalité entre propriété et contrôle des sociétés européennes cotées : étude externe » $n^{\circ}$ MARKT/2006/15/F. Tous les documents mobilisés ici sont disponibles à l'adresse suivante : http://ec.europa.eu/internal_market/company/shareholders/indexb_fr.htm.
} 
Morin [1974, 1994] à la suite de Berle et Means. Toutefois, comme le précisent les auteurs de The Modern Corporation, d'autres logiques que celle du vote en assemblée générale peuvent entrer en compte dans la détermination de la composition des conseils. Une société fortement endettée auprès d'une banque peut en effet lui laisser un ou plusieurs sièges dans l'organe central de gouvernance des firmes. L'analyse menée ici dans le cadre de la mesure du droit des actionnaires ne se focalise néanmoins que sur les déterminants juridiques de la composition du conseil d'administration.

Il convient par ailleurs de noter qu'il existe des définitions plus larges du contrôle qui comprennent notamment les droits de contrôle résiduels sur le capital humain, par définition inaliénable. Si l'on suit Gibbons [2005], et sa lecture des théories de Williamson et de la théorie des droits de propriété de Grossman, Hart et Moore, le contrôle peut alors se définir comme l'espace d'action d'un agent, c'est-à-dire comme le droit de décision que lui confère la possession d'un actif humain ou non humain.

La définition retenue ici est plus restrictive en ce qu'elle se focalise sur les seuls actifs non humains. En effet, le groupe d'administrateurs et/ou d'actionnaires qui parvient à rassembler une majorité en AG, soit le "groupe de contrôle " au sens de Berle et Means, choisit les membres du conseil qui nomment à leur tour les dirigeants de la firme. Ces derniers disposent in fine des droits de contrôle résiduels sur les actifs aliénables de la société. Par extension, et pour indiquer cette filiation, certains auteurs comme Hart [1995] attribuent le contrôle ultime sur les actifs matériels et immatériels de la société au groupe qui, de manière ultime, a nommé les managers. Enfin, les droits de structure construits ici mettent quant à eux l'accent sur les droits de contrôle non résiduels, c'est-à-dire sur les décisions spécifiées par contrat sur lesquelles le droit impose explicitement un vote actionnarial.

La présentation suivante du processus de codage se focalise essentiellement sur les droits de contrôle mais les scores finaux sont reportés pour l'ensemble des mesures. Ainsi, la méthodologie générale est tout d'abord présentée, l'élaboration de l'indicateur des droits de contrôle est alors détaillée et celui-ci fait finalement l'objet d'une description illustrative. L'indicateur des droits de structure et la mesure synthétique du «pouvoir actionnarial » sont alors brièvement décrits pour être enfin comparés aux indicateurs de LLSV [1998] et DLLS [2008].

\section{Méthodologie : forces et faiblesses des indicateurs du pouvoir actionnarial}

La construction d'indicateurs visant à capturer les droits de contrôle et de structure n’échappe pas à l'ensemble des critiques méthodologiques qui sont généralement avancées à l'égard de ce type de mesures. Il convient donc d'en présenter d'abord les faiblesses et la manière dont elles sont ici traitées ${ }^{9}$.

L’un des principaux problèmes dans l'élaboration d'indicateurs tient au coût de la collecte d’informations. Un protocole de codage rigoureux nécessite en effet l’implication des juristes à deux niveaux - même si leurs représentations ne garantissent pas l'exhaustivité de la mesure finale $^{10}$. La mobilisation des firmes d'avocats se situe d'abord sur le plan du recueil des textes

\footnotetext{
${ }^{9}$ L’ensemble du codage proposé ici est spécifique à cet article. L’étude de Shearman \& Sterling LLP [2007] donne des réponses binaires à un ensemble de questions stylisées qui sont ici agrégées dans des indicateurs.

${ }^{10}$ Sur le poids de la représentation des experts dans l'élaboration des indicateurs, cf. Kirat [2006]. Notons à ce sujet que la mobilisation des experts permet d'éviter des erreurs dans le processus de codage, mais n'en n'est pas exempte pour autant. Ainsi, les avocats interrogés par Pagano et Volpin [2005] notent huit erreurs dans l'indicateur original de LLSV, alors que Spamann [2010] en relève un nombre bien supérieur. Martynova et
} 
juridiques au regard d'un problème particulier (une transaction avec apparentés dans le cas de DLLS, ou la nomination et la révocation des administrateurs en ce qui concerne la présente étude). Vient ensuite l'élaboration de la mesure qui requiert un retour des juristes sur la validité du codage. Quelles sont donc les forces et les faiblesses de la mesure des droits de contrôle et de structure au regard du protocole standard d'élaboration des indicateurs juridiques, alors que nous n'avons pu nous-même entrer en interaction avec les professionnels du droit, mais ne faisons ici que mobiliser leur production finale ?

Sur le premier point, et a fortiori sur le second, notons déjà que LLSV [1998] n’ont pas suivi ce protocole dans le choix et le recueil de leurs variables. Les nombreuses critiques à cet égard les ont donc conduits à reconstruire leur indicateur de manière plus rigoureuse. Toujours est-il que si les mesures des droits anti-administrateur de DLLS [2008], Spamann [2010] et Pagano et Volpin [2005], impliquent le concours des juristes dans leur élaboration, elles n'en demeurent pas moins une collection de variables ad hoc et incomplètes. Celles-ci ne capturent, en effet, qu'une partie des dispositifs juridiques visant à protéger les actionnaires minoritaires sans autres justifications que ce dénominateur commun. L'indicateur antimalversation de DLLS est en ce sens plus abouti ${ }^{11}$.

L'indicateur des droits de contrôle n'échappe pas au problème de l'exhaustivité, auquel tentent de remédier les approches fonctionnelles présentées ci-dessus, mais pour un nombre plus restreint de pays (Lele et Siems [2007]). Les droits de contrôle sont construits sur une échelle allant de 0 à 6 , comme l'indicateur anti-administrateur, et ne capturent pas l'ensemble des dispositifs juridiques relatifs à la nomination et la révocation des membres d'un conseil d'administration, essentiellement pour deux raisons. Tout d'abord, il existe une multitude de dispositifs juridiques non pris en compte dans l'étude de droit comparé mobilisée ici, par exemple l'existence de "parachutes dorés » pour les administrateurs. Ensuite, un certain nombre de règles analysées par Shearman \& Sterling LLP [2007] ont dû être rejetées en raison de l'ambiguïté qui les caractérise pour certains pays. L'accès au seul document final et l'impossibilité de lever l'ambiguïté par la lecture détaillée de l'étude invitent à ne pas tenir compte de certaines règles. La mesure finale proposée ne présente donc qu'une indication sur les dispositifs juridiques du contrôle, au risque d'être incomplète et biaisée comme le sont les indicateurs les plus couramment utilisés.

La mesure des droits de contrôle ne peut néanmoins être considérée comme ad hoc, au sens où la définition retenue du contrôle correspond parfaitement à l'un des objets d'analyse de l'étude de droit comparé employée pour l'élaboration de cette mesure. Les correspondants de la firme d'avocats dans chaque pays ont en effet répondu à un questionnaire précis de leur direction consistant à détailler les textes en vigueur sur un certain nombre de dispositifs portant sur la nomination et la révocation des administrateurs. Le problème posé ici, celui de la capacité des actionnaires à nommer ou révoquer les membres d'un conseil d'administration, se trouve donc saisi par l'un des objets de l'étude de droit comparé. Enfin, à l'instar de DLLS [2008] qui se basent sur la présentation stylisée d'une transaction entre apparentés pour

Renneboog [2011] soulignent également l'influence de l'interprétation et de la confusion parmi les juristes euxmêmes.

${ }^{11}$ Pour leur indicateur anti-malversation DLLS affinent leur processus de codage par rapport à la méthode de LLSV qui n'impliquait pas de juristes. DLLS procèdent (i) à l'envoi à des cabinets d'avocats d'un questionnaire fondé sur une transaction stylisée de transferts d'actifs ; (ii) au codage des variables en fonction des réponses reçues et des textes de loi associés ; (iii) à des conférences téléphoniques avec les juristes pour confirmer leur interprétation des réponses reçues et leur codage. 
construire leur indicateur anti-malversation, cette étude décrit les règles juridiques au regard de cas stylisés afin de comparer, par exemple, le type de majorité requis en $\mathrm{AG}^{12}$.

L'indicateur des droits de structure est quant à lui une collection de variables touchant à des décisions sur l'actif et le passif des sociétés ainsi qu’à leurs statuts. Ces variables sont mobilisées afin de rapporter une information supplémentaire sur l’intensité du pouvoir général des actionnaires en AG au travers des diverses juridictions. L’agrégation de ces décisions au sein d'un même indicateur se justifie car elles ont pour point commun de requérir l'expression d'une majorité qualifiée dans au moins un des pays étudiés, ce qui permet d'introduire une variance dans le niveau des votes nécessaires à la maîtrise de la décision.

Reste alors la seconde étape à laquelle nous n'avons pu procéder, à savoir l'interaction avec les professionnels du droit afin d'élaborer le codage final et d'en vérifier la validité. Ce problème est néanmoins surmonté de manière partielle par la rigueur de l'étude de droit comparé. Dans un premier temps, lors de l'élaboration du questionnaire, plusieurs échanges entre la firme d'avocats et ses conseils locaux dans les diverses juridictions ont conduit à la clarification des questions afin d'assurer la cohérence des réponses, procédure que suivent également DLLS [2008] dans l'élaboration de leur indicateur anti-malversation. Pour chaque pays, les réponses sont ensuite détaillées de manière écrite et de façon plus synthétique sous la forme d'un questionnaire à choix multiples, avec la référence des textes de droit mobilisés. Enfin, un document rassemble l'information dans des tableaux de synthèse qui permettent de lever l'ambiguïté des réponses écrites dans le codage final ${ }^{13}$. Toutefois, le nombre de notes infrapaginales - pas moins d'une cinquantaine - accompagnant ces tableaux témoigne du caractère ambigu que peuvent prendre les réponses pour certains dispositifs. Malgré tout, l'ensemble des informations qui sont utilisées, et la manière dont elles sont exploitées dans cet article, sont publiquement disponibles, ce qui est un des critères essentiels pour procéder au codage et rendre possible sa réfutation (Spamann [2006], Lele et Siems [2007]; Siems [2008]).

Une dernière critique porte sur la pondération à accorder à chaque composante de l'indicateur. La procédure retenue ici ne peut faire mieux que ce qui se fait en la matière, à savoir suivre l'hypothèse de l'équivalence entre chaque dispositif. Les diverses méthodes existantes sont appliquées pour le codage final. Elles consistent à sommer chacune des composantes ou à en faire la moyenne. Enfin, pour contrer le scepticisme à l'égard du codage employé, les scores factoriels des analyses en composantes principales sont utilisés comme mesure alternative, à la manière de DLLS [2008], ce qui ne modifie pas la qualité des résultats.

\footnotetext{
12 Pour l'analyse du type de majorité, l'étude fait l'hypothèse suivante : (a) il y a 100 actions votantes dans la société ; (b) 80 actions votantes sont présentes ou représentées durant l’AG ; (c) sur une résolution spécifique durant l'AG, 5 actions votantes ne participent pas au vote, 5 actions votantes expriment un vote blanc, et les 70 actions votantes restantes votent soit oui soit non. Dans ce cadre là, (i) une « majorité simple » requiert 36 votes « oui » (c'est-à-dire 50\% de 70 voix plus un vote); (ii) une «majorité améliorée » requiert 41 votes « oui » (c'est-à-dire $50 \%$ de 80 voix plus un vote); (iii) et une « majorité absolue » requiert 51 votes (c'est-à-dire $50 \%$ de 100 voix plus un vote). Cf. p. 7-8 de la méthodologie de l'étude de Shearman \& Sterling LLP [2007], annexe A. Pour les 15 pays étudiés ici la règle de la majorité absolue n’a pas cours dans les assemblées générales.

${ }^{13}$ Pour ce qui concerne l'indicateur des droits de contrôle, il s'agit essentiellement des tableaux disponibles dans l'annexe B de l'étude de Shearman \& Sterling LLP [2007], p. 134-137 pour la procédure de nomination, et p. 138-142 pour le processus de révocation. Pour l'indicateur des droits de structure, les tableaux des pages 146149 sont mobilisés.
} 


\section{L’indicateur des droits de contrôle}

L'indicateur des droits de contrôle comprend deux variables relatives à la nomination des administrateurs, trois autres portant sur leur révocation et une sixième sur le quorum requis en AG pour nommer et révoquer les administrateurs. La définition de ces variables est donnée dans le tableau A.1.1. (annexe A1) et le codage final est présenté dans le tableau A.1.2. (annexe A1). Trois des variables sur la nomination et la révocation des administrateurs sont de même nature (type de la majorité et quorum). Les trois autres sont spécifiques à l'une des deux procédures. Le principe du codage consiste à améliorer le score en fonction de l'implication croissante des actionnaires dans la nomination et la révocation des administrateurs. Cette implication croissante est notamment capturée par la proportion de voix nécessaire à la ratification d'une décision. Il est certes plus difficile de démettre un administrateur lorsque davantage de voix sont requises. Toutefois, cela induit également un engagement patrimonial plus important de la part des porteurs de titres si ces derniers veulent contrôler la décision. C'est ainsi la place accordée à la participation des grands actionnaires dans la vie de la société qui est mesurée.

L'indicateur capture dans un premier temps le type de la majorité nécessaire en AG pour nommer ou révoquer les administrateurs. Il est ici nécessaire d’établir une variable spécifique à la nomination et l'autre à la révocation puisque la majorité requise n'est pas nécessairement la même dans les deux cas. Elle impose notamment une implication plus importante des actionnaires pour la révocation en Belgique, en Hongrie et au Luxembourg - où la règle de la majorité pour la révocation est une règle obligatoire, contrairement à la majorité requise pour la nomination. Une « majorité améliorée » vaut alors plus qu'une " majorité simple » (cf. note de bas de page $\mathrm{n}^{\circ} 12$ pour la définition des majorités). Par ailleurs, on améliore le score de ces variables si la majorité requise est une règle obligatoire. Une règle par défaut est en effet modifiable dans les statuts de la société, à la hausse comme à la baisse, alors que la règle obligatoire est impérative. On suit en ce sens Spamann [2006] qui montre que les résultats de LLSV [1998] et, notamment, la supériorité du droit des pays de common law, sont substantiellement modifiés dès lors que cette nuance est intégrée dans les indicateurs.

Inversement, il n'est construit qu'une seule variable relative au quorum requis en AG lors de la première convocation, dans la mesure où celui-ci est identique pour la nomination et la révocation dans chacun des pays étudiés ici. Le seuil retenu pour améliorer le score de cette variable est celui de la médiane des pays analysés, soit 20\% des actions votantes.

Une autre variable relative à la nomination des membres du conseil d'administration est introduite. La valeur de celle-ci augmente s’il existe un mécanisme, comme le vote cumulatif, assurant la représentation des actionnaires minoritaires au conseil. Cette mesure est redondante avec l'une des composantes de l'indicateur des droits anti-administrateur qui sera mobilisé dans la suite de cette étude. Il convient toutefois de l'intégrer ici puisqu'elle porte sur la composition du conseil et que son codage prend en compte son caractère contraignant, contrairement à DLLS. Une information supplémentaire est donc ajoutée. La variable prend une valeur unitaire si la représentation des actionnaires minoritaires est obligatoire. Si l'existence d'un mécanisme permettant une telle représentation nécessite une inscription statutaire, alors le score vaut $1 / 2$ et devient nul autrement. L'inscription dans les statuts est en effet relativement contraignante dans la mesure où, une fois adoptée, son retrait requiert un vote en assemblée générale. La corrélation entre cette variable et celle de l'indicateur révisé de DLLS est de 0,42.

Deux autres variables traitent plus spécifiquement de la fermeté de la révocation. La première porte sur la possibilité de voter une telle résolution sans que celle-ci ait nécessité 
l'inscription à l'ordre du jour de l'AG, faisant ainsi de la révocation une décision potentiellement impromptue. La seconde vise à capturer le caractère ad nutum de la révocation, ce qui correspond à un renvoi sans motifs, sans préavis et sans indemnités ${ }^{14}$. Dans la plupart des pays, les administrateurs ne sont pas en effet considérés comme des employés de la société mais comme les mandataires du capital et ne sont donc pas soumis aux règles du droit du travail. Toutefois, certaines juridictions tendent à limiter la rigueur de ce dispositif, contraignant ainsi la discrétion des actionnaires dans la révocation des administrateurs. Cette variable vise à saisir cette gradation de la révocation.

Le pouvoir des actionnaires peut, par ailleurs, être limité par la possibilité pour les administrateurs d'établir une convention d'emploi avec la société, parallèlement à leur activité d'administrateur. C'est par exemple souvent le cas dans les sociétés cotées au Royaume-Uni. Si cette information est disponible dans l'étude de Shearman \& Sterling LLP [2007], elle ne peut être ici mobilisée en raison de l'ambiguïté qui la caractérise. Seule une indication partielle sur la procédure de révocation peut donc être fournie. De même, on sait que le caractère ad nutum de celle-ci sert souvent de justification à la pratique des rémunérations différées, plus communément connue sous l'appellation des parachutes dorés. L'étude de droit comparé ne fournit cependant pas de renseignements à cet égard. Aussi, il n'est pas possible ici d'évaluer, au travers des différents pays, l'ampleur de ce dispositif, d'ailleurs le plus souvent considéré comme un moyen d'enrichissement (Aglietta, Rebérioux [2004]; Petit [2006] ; Llense [2009]) que comme un substitut à la protection sociale des dirigeants.

L’indicateur des droits de contrôle est alors la somme ou la moyenne arithmétique, par pays, des six variables qui viennent d’être présentées.

\section{La régulation du contrôle au travers des pays}

Deux exemples polaires permettent d'illustrer les indicateurs juridiques et l'approche empirique de cette étude. Sur une échelle de 0 à 6, la valeur des droits de contrôle est nulle pour le Royaume-Uni et atteint un score maximal de 5 pour l'Espagne ou la France. Typiquement, la régulation des droits de contrôle s'effectue en faveur des managers ou des administrateurs indépendants au Royaume-Uni et en faveur des actionnaires de contrôle en France ou en Espagne.

Au Royaume-Uni, en vertu des principes contractuels, les statuts d'une société peuvent modifier les règles de nomination et de révocation des administrateurs. Néanmoins, la Companies Act de 1985 intègre pour ce processus un certain nombre de règles obligatoires ${ }^{15}$.

Dans cette juridiction, la nomination et la révocation des administrateurs requièrent une majorité simple. Il s’agit d’une règle par défaut qui peut donc être modifiable statutairement.

\footnotetext{
${ }^{14}$ Cette variable peut faire l'objet d'une comparaison avec une variable similaire élaborée par Lele et Siems [2007] et détaillée par Siems [2008] pour 20 pays à travers le monde. Sur les 10 variables qu'il analyse, il s’agit de la sixième. Sept pays sont communs à son étude et au présent article. La définition de leur variable est légèrement différente car elle prend d'autres éléments en compte. Pour six pays (Allemagne, Espagne, France, Italie, Pays-Bas, Suède), le coefficient de corrélation entre ces deux mesures est de 0,92. En revanche, une différence sensible se note pour le Royaume-Uni qui obtient un score élevé dans l'étude de Siems et la valeur la plus faible ici. L’étude de Shearman \& Sterling LLP [2007] et le protocole de codage précédemment décrit ne permettent pas de modifier cette variable. Toutefois, lorsque la valeur la plus élevée lui est affectée cela ne modifie pas les résultats puisque le Royaume-Uni a toujours les droits de contrôle les plus faibles.

${ }^{15}$ Il convient de noter que le droit des sociétés appartient au régime codifié de l' «equity law ». Celui-ci se démarque donc du "common law» et de son adaptabilité supposée que DLLS [2008] placent au cœur de l'efficacité juridique, même lorsqu'ils décrivent le droit des actionnaires.
} 
La société peut certes soumettre aux actionnaires la possibilité d'améliorer la majorité en instaurant une règle absolue et non plus relative. Néanmoins, elle peut également requérir une majorité qualifiée par exemple au $1 / 3$, ce qui peut amoindrir le poids de la décision actionnariale dans le choix de la composition du conseil d'administration. De plus, le quorum requis pour nommer et révoquer les administrateurs n'est que de deux actionnaires, indépendamment de la fraction du capital qu'ils détiennent. La ratification des résolutions portant sur la nomination et la révocation des administrateurs ne nécessite donc pas une implication massive du vote actionnarial. On peut donc supposer que des administrateurs indépendants ou managers de la société - parviennent plus facilement sous cette juridiction que dans d'autres, à assurer leur cooptation.

De plus, si l'on fait l'hypothèse qu'il n'existe pas d'actionnaire de référence présent ou représenté au conseil, la composition de celui-ci peut être davantage marquée par la présence de managers ou d'administrateurs indépendants dans la mesure où aucun dispositif de représentation des actionnaires minoritaires n’est obligatoire. Et si une société privilégie la présence d'actionnaires minoritaires au conseil, celle-ci n’implique pas nécessairement une inscription statutaire et peut donc être renversée à tout moment. Le contrôle du conseil, dans les situations de forte dispersion actionnariale, dépend alors de la discrétion managériale et de la concurrence sur le marché du travail des administrateurs indépendants - qui sont in fine sélectionnés par le conseil lui-même (Tunc [1994]).

Par ailleurs, selon la section 303 de la Companies Act, les administrateurs sont révocables sans motif mais peuvent bénéficier d'un préavis et d'une indemnité. Ils disposent aussi de l'opportunité de se représenter lors de la séance concernée - ce dernier point n'étant pas saisi dans l'indicateur. Qui plus est, leur révocation nécessite une inscription à l'ordre du jour de l'assemblée générale, ce qui accompagne la logique du préavis dans la mesure où l'ordre du jour est rédigé lors de la convocation de la séance. Enfin, il convient de rappeler que ne sont prises en compte ici que les dispositions réglementaires et non les codes de bonne conduite qui se sont développés sous l'implication conjointe des administrateurs et des investisseurs institutionnels. Ainsi, pour le Royaume-Uni, le rapport Cadbury et la pratique des experts suggèrent que les sociétés cotées requièrent généralement de leurs administrateurs qu'ils se retirent et soumettent d'eux-mêmes leur réélection tous les trois ans (Tunc [1994] ; Shearman \& Sterling LLP [2007]).

En ce qui concerne les droits de contrôle, la régulation britannique consacre donc un pouvoir relativement important aux administrateurs eux-mêmes, au regard du droit dans les autres juridictions. Cela ne signifie pas que le pouvoir des actionnaires soit minoré, comme le montrera l'analyse conjointe des droits de contrôle et de la protection juridique des actionnaires minoritaires. Cela signifie seulement que sur le seul processus de nomination et de révocation des administrateurs, ces derniers disposent d'une protection et d'une discrétion plus importante face aux actionnaires relativement à d'autres pays.

Inversement, la France et l'Espagne disposent de droits de contrôle davantage favorable aux actionnaires. Parmi les quinze juridictions étudiées, la France accorde le plus de poids aux actionnaires dans le processus de nomination et de révocation des administrateurs. La majorité requise en assemblée générale est en effet une «majorité améliorée » et c'est par ailleurs le seul pays où cette majorité est obligatoire pour la procédure de nomination. Aucune disposition statutaire ne peut donc altérer cette règle. Les mêmes dispositifs valent pour la révocation. De plus, le quorum nécessaire pour nommer et révoquer les administrateurs est de $20 \%$ des actions votantes. Autrement dit, le contrôle des sociétés cotées françaises peut être obtenu avec la détention de plus de $10 \%$ des actions, si aucun autre actionnaire ne vient concurrencer ce vote et que le quorum est atteint. Dans les plus grandes sociétés de capitaux, le seuil de $10 \%$ est déjà un seuil non négligeable. 
Toutefois, comme au Royaume-Uni, la France n'impose pas d'obligation sur la représentation des actionnaires minoritaires et ne requiert pas non plus une inscription statutaire pour l'exercice d'une telle pratique, même si les sociétés ont la possibilité de pouvoir le faire.

La procédure de révocation, en revanche, s'effectue davantage à la défaveur des administrateurs et les soumet donc à une plus grande discrétion de la part des actionnaires de contrôle. La révocabilité ad nutum, sans motif, sans préavis et sans indemnités, est en effet une des caractéristiques de la procédure de révocation française. Par ailleurs, l'article L. 225105 du Code de commerce stipule que «l'assemblée ne peut délibérer sur une question qui n’est pas inscrite à l'ordre du jour. Néanmoins, elle peut, en toutes circonstances, révoquer un ou plusieurs administrateurs ou membres du conseil de surveillance et procéder à leur remplacement. » Ainsi, la révocation ne requiert pas une inscription à l'ordre du jour lors de la rédaction de la convocation de l'AG et peut intervenir à n'importe quel moment. Cette règle est un reliquat de l'ancienne jurisprudence des « incidents de séance » qui considère que des circonstances exceptionnelles peuvent intervenir après la convocation ou durant la séance.

La régulation du contrôle consacre donc la décision actionnariale dans certains pays et tend à favoriser le pouvoir des administrateurs relativement aux actionnaires dans d'autres.

\section{La mesure synthétique du pouvoir actionnarial}

Un indicateur des droits de structure vient compléter la mesure des droits de contrôle. Il traduit l'intensité du vote actionnarial requise par chacune des juridictions sur 11 décisions affectant la structure de la société de capitaux. Cinq des décisions portent sur la structure du capital, trois autres sur la structure des actifs et les trois dernières sur la structure sociale (objet, nationalité et statuts de la société). Pour chacune des 11 décisions décrites dans le tableau A.1.1. (annexe A1), on attribue une valeur nulle si aucun vote des actionnaires n'est sollicité, on ajoute $1 / 2$ point si la décision se prend à la majorité simple et un point si elle requiert une majorité qualifiée au $2 / 3$ au moins. Cette mesure, allant potentiellement de 0 à 11 , a une valeur minimale de 4,5 pour le Royaume-Uni et une valeur maximale de 11 pour la Belgique et le Danemark. Sur l'ensemble des pays, 73\% des décisions requièrent une super majorité, $10 \%$ d'entre elles ne nécessitent qu'une majorité simple et les $17 \%$ restants ne font pas appel à un vote en assemblée générale.

La moyenne des droits de contrôle et de structure permet de dégager un indicateur synthétique du pouvoir actionnarial. Les valeurs de l'ensemble des indicateurs sont reportées dans le tableau A.1.3. (annexe A1).

La figure suivante projette les 15 pays étudiés sur un plan composé par l'indicateur du pouvoir actionnarial et par l'indicateur de la protection des minoritaires, synthétisant les mesures anti-administrateur et anti-malversation des principaux auteurs de la Law and Finance. On constate une corrélation négative entre les deux types de mesures indiquant que les pays où les droits de contrôle et de structure sont faibles sont marqués par une forte protection des actionnaires minoritaires et inversement. Il semble plus précisément se dégager au moins trois groupes : l’Irlande et le Royaume-Uni sont caractérisés par une forte régulation des conflits d'agence mais par un faible pouvoir actionnarial. Cinq pays présentent un niveau plutôt intermédiaire sur les deux types d'indicateurs et cinq autres sont marqués par un niveau élevé du pouvoir actionnarial mais par une protection relativement faible des minoritaires. Les Pays-Bas, l’Espagne et la France se détachent quelque peu de ces catégories. 
L’interprétation de cette relation négative entre les deux types de mesures est aisée. Plus on se déplace sur la partie basse et droite du graphique, plus le droit limite la discrétion des administrateurs dans la composition du conseil d'administration au profit de la décision actionnariale. De la même manière, plus les pays se rapprochent de cette zone, plus les décisions affectant la structure de la société cotée requièrent l'expression d'une super majorité en assemblée générale. Inversement, plus l'on se situe dans la partie haute et gauche du graphique, plus le droit facilite la possibilité pour les membres du conseil d'assurer leur cooptation en situation de passivité des porteurs de titre. Cela signifie également que plusieurs des décisions portant sur la structure de la société ne requièrent pas de vote en assemblée générale, ou alors à une simple majorité. Mais en contrepartie, dans ces pays, les conditions d'intervention ou de recours des actionnaires minoritaires sont renforcées par rapport aux juridictions dans lesquelles les droits de contrôle et de structure sont élevés.

On sait que les indicateurs classiques de la Law and Finance (LLSV [1998] et DLLS [2008]) présentent une corrélation négative avec les niveaux de concentration actionnariale des sociétés cotées. On peut donc supposer que les indicateurs juridiques du pouvoir actionnarial présentent une relation positive avec la concentration de la propriété des actions. En effet, plus le droit requiert une implication actionnariale dans le processus de décision en assemblée générale, plus il est nécessaire de détenir une part importante du capital pour en maîtriser le contenu. En ce sens, il convient de se pencher sur le pouvoir basique dont disposent les actionnaires afin d'expliquer la concentration actionnariale, et ne pas focaliser ainsi l'explication sur la régulation des conflits d'agence comme le fait la Law and Finance. Les deux questions qui se posent alors sont les suivantes. Dans quelle mesure l'ensemble de ces dispositions juridiques nationales trouvent-elles une correspondance au niveau local de l'actionnariat des sociétés cotées ? Par ailleurs, les indicateurs classiques de la Law and Finance et ceux du pouvoir actionnarial sont-ils substituables ou complémentaires dans l'explication des formes de contrôle de ces sociétés?

Graphique 1. Indicateurs juridiques du pouvoir actionnarial et de la protection des actionnaires minoritaires en Europe en 2006

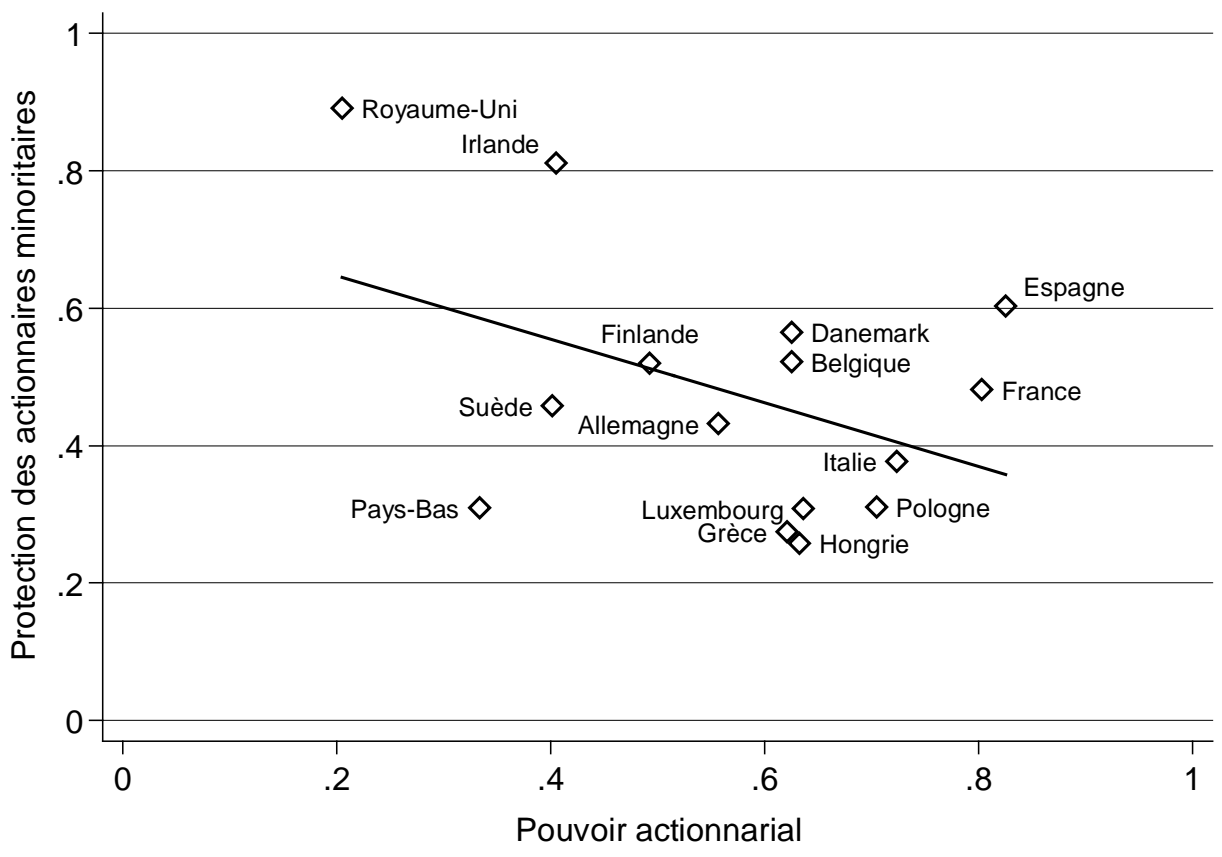


La prochaine section présente l'étude empirique qui met en évidence les complémentarités institutionnelles entre la concentration actionnariale, la régulation des conflits d'agence et le pouvoir décisionnaire que le droit confère aux actionnaires en assemblée générale.

\section{DROIT DES ACTIONNAIRES ET CONCENTRATION ACTIONNARIALE}

Cette partie s’attache à démontrer l'existence d'une complémentarité institutionnelle entre les formes de contrôle des sociétés cotées - de nature actionnariale ou " administratoriale »et les dispositifs juridiques tels que les saisissent les indicateurs présentés précédemment. L'hypothèse à tester et la méthode statistique sont tout d'abord précisées. L'échantillon et les variables de l'analyse sont ensuite présentés. La dernière section décrit les résultats.

\section{Présentation de l’hypothèse à tester et de la méthode}

La présente étude empirique s’attache à soutenir la proposition selon laquelle il existe une complémentarité institutionnelle entre (i) la concentration de l'actionnariat dans les sociétés cotées, (ii) les indicateurs juridiques du pouvoir actionnarial construits ici et (iii) les indicateurs classiques de protection des actionnaires minoritaires. L'hypothèse empirique à tester pour valider l'existence d'une complémentarité institutionnelle est alors la suivante : les différences dans la gradation de la concentration de l'actionnariat, statistiquement significatives entre les pays, s'expliquent par la combinaison des différents indicateurs juridiques et non par l'effet individuel de chacun d'entre eux. Plus précisément, on s'attend à ce qu'une augmentation des indicateurs de la protection des actionnaires minoritaires se traduise par un effet négatif sur la concentration de la propriété des actions et à ce que les indicateurs du pouvoir actionnarial aient un effet positif sur cette variable. Autrement dit, si l'on se réfère au graphique 1 , les niveaux de concentration actionnariale devraient progresser de manière croissante selon la pente négative de la droite qui situe les pays sur le plan des indicateurs juridiques.

Afin de valider cette hypothèse l'étude statistique se décompose en deux temps. Tout d'abord, les indicateurs juridiques et les variables de contrôle définis au niveau national et au niveau local des firmes sont utilisés en tant que variables explicatives dans des régressions par les moindres carrés ordinaires (MCO) visant à expliquer la concentration de la propriété. Les variables juridiques et les grandeurs macroéconomiques constituent des effets fixes par pays qu'il s'agit d'interpréter, une fois contrôlé l'effet individuel de chacune des firmes de l'échantillon. Cette première étape met en évidence une corrélation significative entre la concentration de la propriété et les quatre indicateurs juridiques, pris individuellement ou au travers des indicateurs synthétiques. Les quatre mesures du pouvoir actionnarial et de la protection des minoritaires ont respectivement un effet positif et négatif. Il n'est toutefois pas possible de conclure à l'existence d'une complémentarité institutionnelle sans évaluer l'effet de la combinaison de ces indicateurs sur les formes de contrôle des sociétés cotées.

Pour ce faire, les quatre mesures du droit des actionnaires et leurs multiples combinaisons sont utilisées pour regrouper les pays dans différentes classes en fonction du niveau relativement élevé, intermédiaire ou faible de ces indicateurs. Les groupes ainsi obtenus, par classification ascendante hiérarchique (CAH) sur coordonnées factorielles, sont alors substitués aux indicateurs juridiques dans le modèle linéaire. Un test de Wald sur l'égalité des coefficients de chacune des classes permet enfin d'évaluer la significativité de leurs poids relatifs dans l'explication de l'accroissement de la concentration actionnariale. 
La méthodologie adoptée pour réaliser cette étude combine ainsi les analyses statistiques de plusieurs approches. Le test de la complémentarité institutionnelle repose sur des regroupements de pays effectués sur la base des divers indicateurs juridiques selon la méthode de la CAH employée par les travaux sur la diversité des capitalismes, dite DoC (Amable, [2005]; Berrou et Carrincazeaux [2005] ; Pollin et Vaubourg [2006]). Pour évaluer la corrélation entre ces classes ou les divers indicateurs juridiques et la concentration de la propriété, la procédure d'estimation classique des travaux de la Law and Finance est retenue. Les auteurs de ce courant ont le plus souvent recours à des régressions linéaires par la méthode des moindres carrés ordinaires. Les travaux les plus robustes en la matière sont ceux d'Armour et al. [2009b] ou de Pagano et Volpin [2005]. Puisqu'ils travaillent sur des données de panel, ces auteurs peuvent traiter les effets de l'hétérogénéité inobservable. LLSV [1998] et DLLS [2008] utilisent, quant à eux, des données en coupe et ne peuvent donc procéder à la correction de ces effets. La présente étude, en introduisant des variables de contrôle concernant les firmes, explique davantage d’hétérogénéité que ne le font les principaux auteurs de la Law and Finance. La structure en panel firmes-pays nous permet par ailleurs d'intégrer un effet aléatoire au niveau des juridictions. Tous les résultats présentés ici sont robustes à la prise en compte de cette hétérogénéité inobservable ${ }^{16}$.

\section{Présentation de l'échantillon et des variables à expliquer et explicatives}

L'échantillon d'analyse est constitué par la sélection des plus grandes sociétés de capitaux de 15 pays européens et pour lesquelles des données de marché et une information sur l'actionnariat sont disponibles en 2005 ou 2006. La liste des firmes retenues correspond à celle des sociétés analysées dans l'étude externe menée pour le compte de la Commission Européenne et sur laquelle repose l'élaboration des indicateurs de cet article (cf. p. 97 du rapport final de l'ECGI et al. [2007] pour le détail de l'échantillonnage et p. 117-140 pour la liste des sociétés). Ce rapport retient entre 18 et 20 sociétés par pays pour 15 juridictions européennes, ainsi que 14 sociétés pour l'Estonie. Ces dernières sont ici retirées de l'échantillon par manque d'information sur l'actionnariat et sur les données de marché. Cinq sociétés luxembourgeoises n’ont pu être identifiées et les données de marché pour une autre ne sont pas disponibles. Enfin, la Banque de Grèce, membre de l'Eurosystème, est retirée de l'échantillon dans la mesure où, même si elle est cotée en bourse, la majorité de son conseil est nommé sur décret présidentiel (cf. article 26 de ses statuts) et n’est donc pas soumis à une logique actionnariale.

L'échantillon final est composé de 287 observations expliquant au moins la moitié de la capitalisation boursière de chacun des quinze pays retenus. Au total, 58\% de la capitalisation de l'ensemble des pays sont expliqués comme le montre le tableau A.2.1. (annexe A2). Puisqu'il porte sur les plus grandes sociétés seulement, cet échantillon n'est cependant pas représentatif de l'ensemble du marché boursier.

La variable à expliquer est la nature actionnariale ou " administratoriale » du contrôle des décisions en AG, parmi lesquelles la composition du conseil d'administration. La mesure employée pour évaluer ce contrôle est le pourcentage de la concentration des parts sociales dans les mains d'actionnaires stratégiques. Il s’agit du pourcentage du capital non flottant tel qu'il est évalué périodiquement pour chaque société cotée par les bourses de chaque pays. Il correspond généralement à la part détenue par le premier ou les deux premiers actionnaires. Plus le niveau de la variable expliquée augmente, plus l'on pourra dire qu'un actionnaire ou

\footnotetext{
${ }^{16}$ Ces tests de robustesse non reproduits ici sont disponibles auprès de l'auteur.
} 
un groupe d'actionnaires parvient à contrôler le vote en assemblée générale. La majorité qui s'en dégage, et la composition du conseil d'administration qui en découle, peuvent donc être considérées comme étant de nature actionnariale. Inversement plus le pourcentage de la concentration du capital diminue, plus le contrôle des votes et du conseil pourra être considéré comme étant de nature «administratoriale ». Il est préférable de parler d'un contrôle administratorial, plutôt que managérial, dans la mesure où les administrateurs d'un conseil non contrôlé par des actionnaires peuvent être soit des managers, soit des administrateurs indépendants qui n'exercent pas, par définition, de fonction exécutive dans la société. Les cas dans lesquels les membres d'un conseil ne sont pas issus du management sont fréquents en Irlande et au Royaume-Uni (Kim et al. [2007]). Sur l'ensemble des 287 observations, le pourcentage de la concentration actionnariale est en moyenne de 33\% comme l'indique le tableau A.2.2. (annexe A2).

Les variables explicatives sont à la fois définies au niveau national et au niveau local des sociétés (cf. tableau A.1.1., annexe A1). Les principales variables d'intérêt sont les deux indicateurs juridiques de la protection des actionnaires minoritaires, ainsi que les deux indicateurs du pouvoir actionnarial. Les variables de contrôle introduites sont celles qui, selon Holderness [2008], font l'unanimité dans les études sur les déterminants juridiques de la concentration actionnariale. En ce qui concerne les pays, il convient d'intégrer une grandeur macroéconomique qu'est le PIB par habitant ainsi qu'une variable portant sur l'efficacité économique des tribunaux à exécuter le droit. D’après Holderness [2008], la variable la plus aboutie en la matière est un indicateur développé par Djankov et al. [2003] et qu'ils utilisent ensuite systématiquement dans leurs régressions (Djankov et al. [2008]). Il s'agit d'une mesure de l'efficacité des tribunaux à résoudre un conflit commercial, en l'occurrence la durée de recouvrement d'un chèque sans provisions.

Au moins deux variables de contrôle relatives aux firmes doivent aussi être intégrées, cette fois selon le consensus académique des études portant sur les structures de propriété (Holderness [2008]). L'une porte sur l'âge et l'autre sur la taille de la société. La capitalisation de marché est généralement utilisée pour capturer le poids économique, c’est donc cette mesure qui est retenue ici. Par ailleurs, puisqu'il n'a pas été possible d'obtenir l'âge des sociétés, on utilise le nombre d'années de cotation en bourse. Cette variable apporte peut-être davantage d'informations que la seule date d'incorporation qui permet, quant à elle, de tenir compte de la dilution du capital par héritages successifs. Une société peut en effet exister depuis plusieurs décennies mais n’être cotée que depuis quelques années, si bien que son capital n’a pas encore été dilué par les diverses émissions d'actions. Ces deux variables devraient avoir un effet négatif sur la concentration de la propriété.

Il est nécessaire d'introduire ces variables de contrôle au niveau des sociétés cotées, même si les auteurs de la théorie de l’origine légale les omettent systématiquement dans les régressions linéaires, ou en raison de l'utilisation de tests de moyenne. En effet, alors qu'ils utilisent des mesures de concentration de la propriété qui se définissent au niveau local des firmes, LLSV [1998] et DLLS [2008] ne contrôlent pas les effets liés à la spécificité de ces sociétés, comme leur poids économique. Leur échantillon porte cependant sur plus d'une quarantaine de pays, dans lesquels les plus grandes sociétés peuvent être de taille sensiblement différente, comme le suggère la comparaison des valeurs maximales du tableau A.2.2. (annexe A2) sur une quinzaine de pays seulement. Holderness [2008] relève d'ailleurs de façon critique qu'aucune des neuf études qu'il identifie et qui portent sur les déterminants juridico-économiques de la concentration actionnariale ne procèdent à ce contrôle local.

Ces considérations pratiques sont également chargées théoriquement de sens. Ne pas introduire de variables de contrôle relatives aux firmes revient en effet à suggérer que la concentration de la propriété des actions est uniquement déterminée par des facteurs 
nationaux synthétisés dans des indicateurs juridiques (Vilanova [2007]). Selon cette logique, toutes les sociétés d'un pays devraient être marquées par les mêmes attributs. Par l'introduction de variables de contrôle au niveau des firmes, on admet que l'actionnariat ne s'explique pas seulement par le droit mais est également le résultat de décisions échappant au cadre juridique.

D’autres variables de contrôle sont intégrées pour améliorer la robustesse. Il s'agit tout d'abord de la capitalisation de marché rapportée au PIB. Cette variable constitue pour LLSV [1998] et DLLS [2008] une variable à expliquer. Il est toutefois possible de l'intégrer en tant qu'explicative dans la mesure où l'occurrence de sociétés au capital dispersé est sans doute plus fréquente dans les pays où les marchés financiers sont développés. C’est du moins ce que laisse suggérer l'utilisation par LLSV et DLLS de la concentration actionnariale comme un indicateur de ce développement. En ce qui concerne les firmes, une variable muette indiquant l'appartenance de la société aux secteurs de la banque ou de l'assurance est ajoutée. Les travaux sur les structures de propriété en Europe (La Porta et al. [1999] ; Barca et Becht [2001] ; Faccio et Lang [2002]) décrivent en effet des niveaux plus faibles de concentration actionnariale pour les sociétés financières - qui constituent $25 \%$ de l'échantillon.

\section{Résultats}

\section{Régressions linéaires}

Des régressions par les MCO sont réalisées pour expliquer la concentration de l'actionnariat par des effets fixes définis au niveau des pays, tout en contrôlant certains effets individuels des firmes. Le tableau 1 rapporte les résultats pour les quatre indicateurs juridiques et leurs combinaisons, puis pour les scores factoriels obtenus à partir des variables juridiques et macroéconomiques. Tous les modèles présentent des écarts-types robustes à l'hétéroscédasticité et permettent la corrélation des erreurs parmi les firmes de même nationalité. Les indicateurs juridiques et les grandeurs macroéconomiques ne permettent pas en effet de considérer les observations comme indépendantes au sein de chaque pays. Par ailleurs, malgré la multicolinéarité de certaines variables, comme le rapporte la table des corrélations en annexe, les facteurs d'inflation de la variance indiquent pour toutes les régressions que ce n'est pas un problème majeur ${ }^{17}$. D'ailleurs, celui-ci est surmonté par l'utilisation des scores factoriels. Enfin, les résultats du tableau 1 sont robustes au retrait des variables non significatives.

Les corrélations entre chacun des quatre indicateurs juridiques de base et le niveau de la concentration actionnariale sont statistiquement significatives, une fois les variables de contrôle introduites (modèles 1 et 2 puis 4 et 5). Les indicateurs des droits de contrôle et de structure, ainsi que leur combinaison dans l'indicateur du pouvoir actionnarial (modèle 3), présentent les niveaux de significativité les plus importants. Les probabilités associées aux mesures de la protection des minoritaires ne sont inférieures qu'au seuil de $10 \%$, mais leur combinaison (modèle 6) réduit cette marge d'erreur à 5\%. Dans l'ensemble, les mesures juridiques du pouvoir actionnarial sont davantage explicatives que les indicateurs classiques, ce qui n'est pas altéré lors de la prise en compte simultanée de ces deux mesures synthétiques (modèle 7). Puisque tous ces éléments contribuent à expliquer la concentration de la propriété des actions, on peut en déduire que la régulation captée par ces deux types d'indicateurs n'est

\footnotetext{
${ }^{17}$ Les facteurs d’inflation de la variance sont pour chaque variable compris entre 1 et 3 .
} 
Tableau 1. Régulation du contrôle, des conflits d'agence et concentration actionnariale

Ce tableau présente les résultats de neuf régressions par les MCO pour l'échantillon des 287 firmes des 15 pays étudiés. La variable dépendante est le pourcentage de la concentration actionnariale. Les principales variables indépendantes sont : les indicateurs (a) des droits de contrôle, (b) des droits de structure, (c) du pouvoir actionnarial (moyenne arithmétique de (a) et (b)), (d) des droits anti-administrateur, (e) des droits anti-malversation, (f) de la protection des actionnaires minoritaires (moyenne arithmétique de (d) et (e)). Dans le modèle (8), les variables (a), (b), (d), (e), (g), (h), (i) définies au niveau national sont remplacées par les scores factoriels représentant chacune d'entre elles après rotation des axes. Dans le modèle (9), les sept facteurs sont intégrés avant rotation des axes mais seuls les facteurs significatifs sont ici reportés. Les écarts-types robustes sont entre parenthèses. L’autocorrélation des erreurs est permise parmi les sociétés appartenant à un même pays, la présence des indicateurs juridiques et macroéconomiques rendant les observations non indépendantes. Les niveaux de significativité statistique sont indiqués par : ${ }^{*} p<0.10,{ }^{* * *} p<0.05$, ${ }^{* * *} p<0.01$.

\begin{tabular}{|c|c|c|c|c|c|c|c|c|c|}
\hline \multirow{2}{*}{$\begin{array}{l}\text { Dépendante : \% concentration actionnariale } \\
\text { Indépendantes }\end{array}$} & $(1)$ & (2) & \multirow[t]{2}{*}{ (3) } & (4) & (5) & (6) & \multirow[t]{2}{*}{ (7) } & \multicolumn{2}{|l|}{ (8) } \\
\hline & \multicolumn{2}{|c|}{ Pouvoir actionnarial } & & \multicolumn{3}{|c|}{ Protection des minoritaires } & & \multicolumn{2}{|c|}{ Scores factoriels } \\
\hline & $\begin{array}{c}20.511^{* *} \\
(7.175)\end{array}$ & & & & & & & $\begin{array}{c}4.559 * * * \\
(1143)\end{array}$ & \\
\hline (b) Droits de structure & & $\begin{array}{c}35.036 * * * \\
(9.902)\end{array}$ & & & & & & $\begin{array}{c}4.225 * * * \\
(0.690)\end{array}$ & \\
\hline (c) Indicateur du pouvoir actionnarial & & & $\begin{array}{c}40.723^{* * *} \\
(11.561)\end{array}$ & & & & $\begin{array}{c}35.680 * * * \\
(9.992)\end{array}$ & & \\
\hline (d) Droits anti-administrateur & & & & $\begin{array}{c}-29.262 * \\
(14.012)\end{array}$ & & & & $\begin{array}{c}-4.814 * * * \\
(0.744)\end{array}$ & \\
\hline (e) Droits anti-malversation & & & & & $\begin{array}{l}-17.380^{*} \\
(9.022)\end{array}$ & & & $\begin{array}{c}-3.772 * * * \\
(0.750)\end{array}$ & \\
\hline (f) Indicateur de la protection des minoritaires & & & & & & $\begin{array}{c}-26.514 * * \\
(9.370)\end{array}$ & $\begin{array}{c}-17.159 * * \\
(7.393)\end{array}$ & & \\
\hline (g) Ln(temps recouvrement chèque en blanc) & $\begin{array}{c}-0.679 \\
(2.717)\end{array}$ & $\begin{array}{c}-1.351 \\
(3.584)\end{array}$ & $\begin{array}{l}-2.351 \\
(3.094)\end{array}$ & $\begin{array}{l}-0.953 \\
(3.416)\end{array}$ & $\begin{array}{c}1.627 \\
(2.999)\end{array}$ & $\begin{array}{c}0.474 \\
(3.074)\end{array}$ & $\begin{array}{l}-2.567 \\
(2.575)\end{array}$ & $\begin{array}{c}0.692 \\
(1.155)\end{array}$ & \\
\hline (h) $\mathrm{Ln}(\mathrm{PIB} / \mathrm{POP})$ & $\begin{array}{l}-5.557 \\
(6.048)\end{array}$ & $\begin{array}{l}-12.215 \\
(7.377)\end{array}$ & $\begin{array}{l}-8.776 \\
(5.826)\end{array}$ & $\begin{array}{l}-6.554 \\
(5.941)\end{array}$ & $\begin{array}{l}-5.086 \\
(6.353)\end{array}$ & $\begin{array}{l}-5.404 \\
(5.920)\end{array}$ & $\begin{array}{l}-7.685 \\
(5.365)\end{array}$ & $\begin{array}{c}-3.677 * * \\
(1.709)\end{array}$ & \\
\hline (i) Ln(Capitalisation de marché/PIB) & $\begin{array}{c}-0.825 \\
(6.000)\end{array}$ & $\begin{array}{c}4.952 \\
(8.096)\end{array}$ & $\begin{array}{l}2.870 \\
(5.994)\end{array}$ & $\begin{array}{l}-0.010 \\
(7.059)\end{array}$ & $\begin{array}{l}-0.685 \\
(8.117)\end{array}$ & $\begin{array}{l}-0.222 \\
(7.673)\end{array}$ & $\begin{array}{l}3.112 \\
(5.850)\end{array}$ & $\begin{array}{l}-1.186 \\
(1.277)\end{array}$ & \\
\hline $1^{\text {er }}$ facteur - effet pays & & & & & & & & & $\begin{array}{c}3.997 * * * \\
(0.619)\end{array}$ \\
\hline $3^{\mathrm{e}}$ facteur - effet pays & & & & & & & & & $\begin{array}{c}1.787 * * \\
(0.632)\end{array}$ \\
\hline Ln(Capitalisation de marché des firmes) & $\begin{array}{c}-4.463 * * * \\
(0.822)\end{array}$ & $\begin{array}{c}-3.892 * * * \\
(1.151)\end{array}$ & $\begin{array}{c}-4.358^{* * *} \\
(0.695)\end{array}$ & $\begin{array}{c}-3.292 * * * \\
(0.999)\end{array}$ & $\begin{array}{c}-3.702^{* * *} \\
(1.008)\end{array}$ & $\begin{array}{c}-3.437 * * * \\
(0.963)\end{array}$ & $\begin{array}{c}-3.915^{* * *} \\
(0.653)\end{array}$ & $\begin{array}{c}-3.599 * * * \\
(0.641)\end{array}$ & $\begin{array}{c}-3.599 * * * \\
(0.641)\end{array}$ \\
\hline Durée de cotation & $\begin{array}{c}-0.591 * * * \\
(0.171)\end{array}$ & $\begin{array}{c}-0.594 * * * \\
(0.164)\end{array}$ & $\begin{array}{c}-0.570 * * * \\
(0.171)\end{array}$ & $\begin{array}{c}-0.605^{* * *} \\
(0.162)\end{array}$ & $\begin{array}{c}-0.588 * * * \\
(0.168)\end{array}$ & $\begin{array}{c}-0.587 * * * \\
(0.166)\end{array}$ & $\begin{array}{c}-0.549 * * * \\
(0.171)\end{array}$ & $\begin{array}{c}-0.571^{* * *} \\
(0.170)\end{array}$ & $\begin{array}{c}-0.571^{* * * *} \\
(0.170)\end{array}$ \\
\hline Société financière & $\begin{array}{c}-3.085 \\
(3.406)\end{array}$ & $\begin{array}{c}-3.442 \\
(3.325)\end{array}$ & $\begin{array}{l}-2.954 \\
(3.332)\end{array}$ & $\begin{array}{l}-3.954 \\
(3.462)\end{array}$ & $\begin{array}{c}-3.401 \\
(3.462)\end{array}$ & $\begin{array}{c}-3.592 \\
(3.482)\end{array}$ & $\begin{array}{l}-2.989 \\
(3.348)\end{array}$ & $\begin{array}{c}-3.667 \\
(3.255)\end{array}$ & $\begin{array}{l}-3.667 \\
(3.255)\end{array}$ \\
\hline Constante & $\begin{array}{c}142.865 * * \\
(57.746)\end{array}$ & $\begin{array}{c}165.223 * * \\
(77.020) \\
\end{array}$ & $\begin{array}{c}151.527 * * \\
(58.384) \\
\end{array}$ & $\begin{array}{c}164.443 * * \\
(58.055) \\
\end{array}$ & $\begin{array}{c}133.150 * \\
(63.139) \\
\end{array}$ & $\begin{array}{c}143.601^{* *} \\
(57.725) \\
\end{array}$ & $\begin{array}{c}146.981 * * * \\
(48.637) \\
\end{array}$ & $\begin{array}{c}77.420 * * * \\
(4.947) \\
\end{array}$ & $\begin{array}{c}77.420 * * * \\
(4.947) \\
\end{array}$ \\
\hline $\mathrm{R}^{2}$ & 0.32 & 0.32 & 0.34 & 0.31 & 0.30 & 0.31 & 0.35 & 0.38 & 0.38 \\
\hline
\end{tabular}


pas substituable, ou fonctionnellement équivalente ${ }^{18}$. Il n’est toutefois pas encore possible de conclure à leur complémentarité sans évaluer l'effet de leur combinaison sur le niveau de la concentration actionnariale.

Les deux dernières colonnes du tableau 1 présentent les résultats lorsque les coordonnées factorielles des pays sont substituées aux sept variables définies au niveau national. La régression $\mathrm{n}^{\circ} 8$ contient les scores factoriels de l'analyse en composantes principales (ACP) après rotation des axes, ce qui permet d'attribuer une variable à chacune des composantes principales. La régression $\mathrm{n}^{\circ} 9$ présente les résultats avant rotation des axes, lorsque le premier facteur capte l'essentiel de la variance ${ }^{19}$.

\section{Classification ascendante hiérarchique}

Afin de combiner les indicateurs juridiques qui ont un impact de signe opposé sur la variable dépendante, il est possible de procéder à un regroupement des pays en diverses classes en fonction de leur proximité institutionnelle. Les groupes sont obtenus par des classifications ascendantes hiérarchiques $(\mathrm{CAH})$ menées sur les quatre mesures du droit puis sur leurs combinaisons par ACP. Les classifications sont réalisées selon la méthode de Ward pour agréger les pays, et la mesure de la dissimilarité correspond au calcul d'une distance euclidienne. Cette méthode est bien adaptée aux cas d'orthogonalité des axes sur lesquels sont mesurées les coordonnées factorielles (Evrard et al. [2003]). Ces classes seront ensuite substituées aux indicateurs eux-mêmes dans les régressions précédentes. L’intensité des coefficients de chaque groupe de pays pourra alors faire l'objet d'une comparaison statistique grâce à un test de Wald.

Les CAH réalisées sur chacun des indicateurs différencient trois classes reflétant le niveau relativement faible, intermédiaire ou fort de ces mesures légales. La classification des pays sur la base de plusieurs indicateurs se déroule quant à elle en deux temps. Des ACP, non détaillées ici, sont d'abord menées sur différentes combinaisons des variables juridiques. Les coordonnées factorielles des pays servent alors de base aux CAH. Les combinaisons des deux indicateurs du pouvoir actionnarial, ou des deux mesures de la protection des minoritaires, sont elles aussi séparées en trois classes traduisant la gradation des deux types d'indicateurs considérés. Enfin, les CAH prenant en compte les quatre variables juridiques permettent de dégager trois groupes selon les combinaisons suivantes : (i) forte protection des minoritaires et faible pouvoir actionnarial, (ii) niveau intermédiaire des deux indicateurs, puis (iii) faible protection des minoritaires et pouvoir actionnarial élevé. Une telle composition est conforme à la relation négative entre les deux indicateurs synthétiques mise en évidence lors de leur présentation. Cette CAH en trois groupes n'est pas la plus optimale mais sa composition est robuste à diverses méthodes de classification ${ }^{20}$. La CAH en quatre groupes, la plus optimale,

\footnotetext{
18 L'équivalence fonctionnelle pourrait ici se comprendre de la manière suivante : afin de favoriser une dispersion du capital des sociétés cotées, il faudrait indifféremment soit diminuer les droits de contrôle et de structure, soit augmenter les mesures de la protection des actionnaires minoritaires. La significativité de l'un des indicateurs disparaîtrait alors au profit de l'autre. Les problématiques captées par chacun des indicateurs sont en ce sens bien distinctes. L'une se focalise sur la capacité d'intervention des minoritaires, l'autre sur le pouvoir décisionnel des actionnaires.

${ }^{19}$ Tous les facteurs sont intégrés dans la régression mais seul le premier et le troisième sont reportés dans le tableau 1 puisqu'ils sont statistiquement significatifs. Le troisième facteur est déterminé à 73\% par les indicateurs des droits de structure et de contrôle. Ceux-ci contribuent à la composition du premier axe à hauteur de $19 \%$, et les deux indicateurs de la protection des actionnaires minoritaires le déterminent à $29 \%$.

${ }^{20}$ La classification en trois groupes ne se modifie pas pour diverses mesures de dissimilarité. Elle ne varie pas non plus lorsque les CAH sont réalisées en pondérant ou non les axes factoriels par leur valeur propre. En
} 
est indiquée mais non analysée ici du fait de la sensibilité de cette classification à la méthode utilisée ${ }^{21}$.

Le tableau 2 présente les groupes obtenus sur la base des divers indicateurs et de leurs combinaisons. Pour les classifications réalisées sur une ou deux variables juridiques, le numéro des classes traduit le niveau des indicateurs (1 pour élevé, 2 pour intermédiaire et 3 pour faible). Pour les regroupements basés sur les quatre indicateurs, le numéro affecté à chaque classe n'a pas de signification particulière.

Tableau 2. Classifications des pays par proximité institutionnelle

\begin{tabular}{|c|c|c|c|c|c|c|c|c|}
\hline CAH réalisée sur & \multicolumn{4}{|c|}{ Un indicateur } & \multicolumn{2}{|c|}{ Deux indicateurs } & \multicolumn{2}{|c|}{ Quatre indicateurs } \\
\hline & 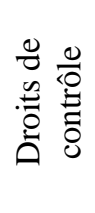 & 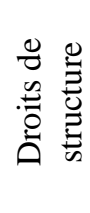 & 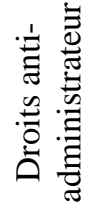 & 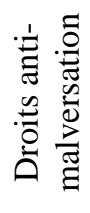 & 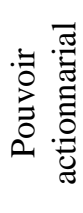 & 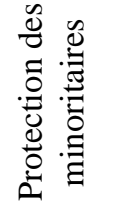 & $\begin{array}{l}\mathscr{U} \\
\mathscr{W} \\
\tilde{\Xi} \\
\tilde{U} \\
m\end{array}$ & $\begin{array}{l}\mathscr{U} \\
\mathscr{W} \\
\tilde{U} \\
\dot{U} \\
\forall\end{array}$ \\
\hline Allemagne & 3 & 1 & 2 & 3 & 2 & 2 & 1 & 1 \\
\hline Belgique & 3 & 1 & 3 & 2 & 2 & 2 & 1 & 1 \\
\hline Danemark & 3 & 1 & 2 & 2 & 2 & 2 & 1 & 1 \\
\hline Finlande & 3 & 1 & 2 & 2 & 2 & 2 & 1 & 1 \\
\hline Pays-Bas & 3 & 3 & 3 & 3 & 3 & 3 & 1 & 1 \\
\hline Suède & 3 & 2 & 2 & 3 & 3 & 2 & 1 & 1 \\
\hline Espagne & 1 & 1 & 1 & 3 & 1 & 2 & 2 & 2 \\
\hline France & 1 & 1 & 2 & 3 & 1 & 2 & 2 & 2 \\
\hline Grèce & 3 & 1 & 3 & 3 & 2 & 3 & 2 & 3 \\
\hline Italie & 2 & 1 & 3 & 2 & 1 & 3 & 2 & 3 \\
\hline Luxembourg & 2 & 1 & 3 & 3 & 1 & 3 & 2 & 3 \\
\hline Hongrie & 2 & 2 & 3 & 3 & 1 & 3 & 2 & 3 \\
\hline Pologne & 2 & 1 & 3 & 3 & 1 & 3 & 2 & 3 \\
\hline Irlande & 3 & 2 & 1 & 1 & 3 & 1 & 3 & 4 \\
\hline Royaume-Uni & 3 & 3 & 1 & 1 & 3 & 1 & 3 & 4 \\
\hline
\end{tabular}

Chaque classification aboutit à un regroupement différent, comme l'illustre l'exemple suivant. Les indicateurs classiques de protection des minoritaires isolent un modèle anglosaxon, conformément aux résultats traditionnels de la Law and Finance. Les indicateurs du pouvoir actionnarial rassemblent quant à eux le Royaume-Uni et l'Irlande avec les Pays-Bas et la Suède. Ces rapprochements sur la base des mesures juridiques trouvent également une justification économique. Les capitalisations de marché de ces quatre pays présentent en effet plusieurs similitudes (Auvray et Granier [2009], p. 83). Elles sont tout d'abord marquées par une prépondérance de gestionnaires pour le compte de tiers. En Suède et au Royaume-Uni, ces investisseurs sont essentiellement nationaux en raison de l'existence d'un régime de retraite par capitalisation développé. En Irlande et aux Pays-Bas, la part nationale des gestionnaires de portefeuille est plutôt faible au profit d'une part états-unienne très élevée. La régulation dans ces pays est orientée dans le sens d'un plus grand pouvoir concédé aux

revanche, le regroupement en quatre classes ne peut être considéré comme robuste car la composition des groupes se modifie dès qu'un changement mineur est introduit. Ainsi, lors du passage de trois à quatre classes, les Pays-Bas se retrouvent isolés avant la France et l’Espagne lorsque la distance euclidienne est élevée au carré.

${ }^{21}$ Pour les CAH des deux dernières colonnes du tableau 2, les variances intra-classe et interclasses se répartissent respectivement dans les proportions suivantes : 50\%-50\% pour la classification en trois groupes, et 36\%-64\% pour la classification en quatre groupes. 
administrateurs, peut-être en raison de la présence d'actionnaires davantage concernés par la gestion de leur portefeuille que par la marche courante des sociétés. La combinaison des indicateurs permet alors de distinguer parmi ces pays, ceux pour lesquels la discrétion administratoriale est soumise à une facilité d'intervention des actionnaires minoritaires.

La classification finale en trois groupes permet de retrouver certaines catégories de la DoC. La première classe, marquée par un niveau intermédiaire des deux types de mesure, regroupe des pays des capitalismes européen continental et social-démocrate. Le deuxième groupe, dominé par de forts indicateurs du pouvoir actionnarial et une faible protection des minoritaires, fait surtout ressortir les pays du modèle méditerranéen et du capitalisme d'Europe Centrale et de l'Est. Enfin, l'Irlande et le Royaume-Uni sont isolés en raison de leur caractère contrasté sur les quatre indicateurs, reflétant par ailleurs leur regroupement dans un capitalisme libéral de marché. En revanche, des classifications plus fines se détachent de ces catégorisations traditionnelles, dans la mesure où ne sont ici pris en compte qu'un petit nombre d'indicateurs juridiques. Par définition, une analyse de la diversité des capitalismes et des complémentarités institutionnelles qui les caractérisent devrait simultanément prendre en compte l'ensemble des secteurs institutionnels selon la méthodologie initiée par Amable [2005], ce qui n'est pas l'objet de cet article. Notre apport consiste à mettre en évidence la complémentarité institutionnelle existant entre l'actionnariat des grandes sociétés cotées et la régulation juridique du pouvoir actionnarial et des conflits d'agence.

\section{Test de la complémentarité institutionnelle}

Les groupes obtenus par chaque classification sont maintenant substitués aux indicateurs juridiques dans les régressions réalisées précédemment - c'est-à-dire en conservant les variables de contrôle. Il convient de vérifier si chaque classe a un effet significativement contrasté sur la concentration actionnariale des sociétés cotées. Par exemple, la concentration actionnariale du groupe caractérisé par un pouvoir actionnarial élevé devrait être plus forte que celle des groupes pour lesquels le niveau de cet indicateur est intermédiaire ou faible. De même, les sociétés entrant dans la classe marquée par une forte protection des minoritaires devraient avoir un actionnariat significativement moins concentré que celui des sociétés appartenant à l'un des deux autres groupes. Pour vérifier de telles relations, les classes sont comparées deux à deux selon un test de Wald d'égalité des coefficients. L’hypothèse de la complémentarité institutionnelle à valider est la suivante: les classifications prenant simultanément en compte les deux types d'indicateurs permettent de dégager des classes dont les effets respectifs sont significativement différents les uns des autres. Le tableau 3 rapporte les tests pour les groupes obtenus sur la base de deux ou quatre indicateurs.

Les résultats statistiques permettant de conclure à la complémentarité institutionnelle entre les deux types de mesures juridiques et la concentration de la propriété des actions sont les suivants. Les regroupements menés sur un ou deux des indicateurs ne permettent pas de distinguer trois groupes de pays dont l'effet sur la concentration actionnariale serait significativement différent. Pour chacune de ces classifications, l'hypothèse nulle d'égalité des coefficients entre deux classes ne peut être rejetée dans le cas le plus optimiste qu'avec une marge d'erreur de $14 \%{ }^{22}$. Il se dégage donc toujours au moins deux groupes de pays dont

\footnotetext{
${ }^{22}$ Dans le tableau 3A, les classes pour lesquelles les indicateurs du pouvoir actionnarial sont forts ou intermédiaires n’ont pas des structures de propriété significativement différentes (la marge d'erreur du rejet de l'hypothèse nulle d'égalité des coefficients est de 14,3\%). Dans le tableau 3B, les groupes pour lesquels la protection des minoritaires est faible ou intermédiaire ne sont pas non plus marqués par une différence significative de leur concentration actionnariale (la marge d'erreur du rejet de l'hypothèse nulle est de 27,9\%).
} 
Tableau 3. Test de la complémentarité institutionnelle entre la concentration de l'actionnariat et les indicateurs juridiques

Ce tableau rapporte la statistique $F$ des tests d'égalités des coefficients de régressions par les MCO, ainsi que leurs probabilités associées entre parenthèses. La variable dépendante est le pourcentage de la concentration actionnariale. Les variables indépendantes sont les variables de contrôle détaillées dans le tableau A.1.1 (annexe 1) et un ensemble de variables dichotomiques identifiant les différents groupes issus des classifications ascendantes hiérarchiques (CAH) menées sur les indicateurs juridiques. Les classes correspondent au niveau relatif des indicateurs : faible, intermédiaire ou fort. Les tableaux A, B et C rapportent les tests d'égalité des coefficients des classes issues des CAH respectivement réalisées sur les deux indicateurs du pouvoir actionnarial, les deux indicateurs de la protection des minoritaires puis sur ces quatre indicateurs. Les pourcentages moyens de la concentration actionnariale de chacune des classes sont donnés à titre indicatif. Leur intensité relative reflète celle de leur coefficient. Les chiffres en gras correspondent aux cas de rejet de l'hypothèse nulle d'égalité des coefficients.

Tableau 3A : Pouvoir actionnarial

\begin{tabular}{|c|c|c|c|c|}
\hline $\begin{array}{l}\text { Classe de référence (\% moyen de } \\
\text { sa concentration actionnariale) }\end{array}$ & & Intermédiaire & Fort & $\begin{array}{l}\text { Classe de référence }(=1) \\
\text { contre toutes les autres }(=0)\end{array}$ \\
\hline $\begin{array}{l}\text { Faible } \\
(17.79 \%)\end{array}$ & $\begin{array}{l}\text { Stat. } F_{1,14} \\
(\text { Prob }>F)\end{array}$ & $\begin{array}{c}4.96 \\
(0.0428)\end{array}$ & $\begin{array}{c}19.86 \\
(0.0005)\end{array}$ & $\begin{array}{c}10.94 \\
(0.0052)\end{array}$ \\
\hline $\begin{array}{l}\text { Intermédiaire } \\
(32.39 \%)\end{array}$ & $\begin{array}{l}\text { Stat. } F_{1,14} \\
(\text { Prob }>F)\end{array}$ & & $\begin{array}{c}2.41 \\
(0.1431)\end{array}$ & $\begin{array}{c}0.02 \\
(0.8787)\end{array}$ \\
\hline $\begin{array}{l}\text { Fort } \\
(44.40 \%)\end{array}$ & $\begin{array}{l}\text { Stat. } F_{1,14} \\
(\text { Prob }>F)\end{array}$ & & & $\begin{array}{c}6.79 \\
(0.0208)\end{array}$ \\
\hline $\begin{array}{l}\text { Toutes les classes sont égales à } \\
\text { chacune des autres }\end{array}$ & $\begin{array}{l}\text { Stat. } F_{2,14} \\
(\text { Prob }>F)\end{array}$ & \multicolumn{3}{|c|}{$\begin{array}{c}10.13 \\
(0.0019)\end{array}$} \\
\hline
\end{tabular}

Tableau 3B : Protection des minoritaires

\begin{tabular}{|c|c|c|c|c|}
\hline $\begin{array}{l}\text { Classe de référence (\% moyen de } \\
\text { sa concentration actionnariale) }\end{array}$ & & Intermédiaire & Fort & $\begin{array}{c}\text { Classe de référence }(=1) \\
\text { contre toutes les autres }(=0)\end{array}$ \\
\hline $\begin{array}{l}\text { Faible } \\
(45.37 \%)\end{array}$ & $\begin{array}{l}\text { Stat. } F_{1,14} \\
(\text { Prob }>F)\end{array}$ & $\begin{array}{c}1.27 \\
(0.2787)\end{array}$ & $\begin{array}{c}17.02 \\
(0.0010)\end{array}$ & $\begin{array}{c}2.55 \\
(0.1323)\end{array}$ \\
\hline $\begin{array}{l}\text { Intermédiaire } \\
(29.26 \%)\end{array}$ & $\begin{array}{l}\text { Stat. } F_{1,14} \\
(\text { Prob }>F)\end{array}$ & & $\begin{array}{c}11.65 \\
(0.0042)\end{array}$ & $\begin{array}{c}0.05 \\
(0.8311)\end{array}$ \\
\hline $\begin{array}{l}\text { Fort } \\
(12.41 \%)\end{array}$ & $\begin{array}{l}\text { Stat. } F_{1,14} \\
(\text { Prob }>F)\end{array}$ & & & $\begin{array}{c}19.82 \\
(0.0005)\end{array}$ \\
\hline $\begin{array}{l}\text { Toutes les classes sont égales à } \\
\text { chacune des autres }\end{array}$ & $\begin{array}{l}\text { Stat. } F_{2,14} \\
(\text { Prob }>F)\end{array}$ & \multicolumn{3}{|c|}{$\begin{array}{c}12.12 \\
(0.0009)\end{array}$} \\
\hline
\end{tabular}

Tableau 3C : Pouvoir actionnarial (PA) et Protection des minoritaires (PM)

\begin{tabular}{|c|c|c|c|c|}
\hline $\begin{array}{l}\text { Classe de référence (\% moyen de } \\
\text { sa concentration actionnariale) }\end{array}$ & & $\begin{array}{c}\text { PA et PM } \\
\text { intermédiaires }\end{array}$ & $\begin{array}{l}\text { Fort PA et } \\
\text { faible PM }\end{array}$ & $\begin{array}{c}\text { Classe de référence }(=1) \\
\text { contre toutes les autres }(=0)\end{array}$ \\
\hline $\begin{array}{l}\text { Faible PA et forte PM } \\
(12.41 \%)\end{array}$ & $\begin{array}{l}\text { Stat. } F_{1,14} \\
(\text { Prob }>F)\end{array}$ & $\begin{array}{c}4.04 \\
(0.0641)\end{array}$ & $\begin{array}{c}33.42 \\
(0.0000)\end{array}$ & $\begin{array}{c}19.82 \\
(0.0005)\end{array}$ \\
\hline $\begin{array}{l}\text { PA et PM intermédiaires } \\
(27.78 \%)\end{array}$ & $\begin{array}{l}\text { Stat. } F_{1,14} \\
(\text { Prob }>F)\end{array}$ & & $\begin{array}{c}3.85 \\
(0.0699)\end{array}$ & $\begin{array}{c}0.04 \\
(0.8389)\end{array}$ \\
\hline $\begin{array}{l}\text { Fort PA et faible PM } \\
(44.12 \%)\end{array}$ & $\begin{array}{l}\text { Stat. } F_{1,14} \\
(\text { Prob }>F)\end{array}$ & & & $\begin{array}{c}7.88 \\
(0.0140)\end{array}$ \\
\hline $\begin{array}{l}\text { Toutes les classes sont égales à } \\
\text { chacune des autres }\end{array}$ & $\begin{array}{l}\text { Stat. } F_{2,14} \\
(\text { Prob }>F)\end{array}$ & \multicolumn{3}{|c|}{$\begin{array}{c}20.21 \\
(0.0001)\end{array}$} \\
\hline
\end{tabular}


les plus grandes sociétés cotées ont des niveaux de concentration actionnariale similaire. On notera que de simples tests de moyenne sur la concentration actionnariale des classes des tableaux 3A ou 3B permettent d'identifier des groupes significativement différents. L'introduction de variables de contrôle se justifie donc en ce qu'elle permet d'établir des distinctions entre les pays moins fortuites que celles produites par les tests couramment utilisés par les principaux auteurs de la Law and Finance.

En revanche, les regroupements effectués sur la base de l'ensemble des indicateurs juridiques permettent de différencier des classes dont l'effet sur la concentration actionnariale se contraste de manière significative ${ }^{23}$. Autrement dit, toute l'information contenue dans les quatre indicateurs est suffisamment pertinente pour générer des regroupements de pays conformes aux hypothèses émises sur la cohérence institutionnelle entre la concentration de la propriété, les indicateurs juridiques du pouvoir actionnarial et les indicateurs légaux de la protection des minoritaires.

\section{UNE COMPLEMENTARITE INSTITUTIONNELLE : DROITS DES ACTIONNAIRES ET SELECTION DE LA GOUVERNANCE}

Les trois configurations mises en évidence par l'étude empirique font intervenir des actionnaires de contrôle, des actionnaires minoritaires externes et des administrateurs. Les relations entre ces trois types d'acteurs et leurs préférences, qui se manifestent dans la production de règles juridiques et dans la concentration actionnariale, peuvent être décrites selon la grille de lecture du «canal de l'économie politique » (Roe et Siegel [2009] ; Roe [2006, 2003]) ou encore du « canal institutionnel » (Armour et al. [2009a, b]) de la production du droit ${ }^{24}$. La notion de complémentarité institutionnelle d'Aoki [2001] est au cœur de ces dernières analyses. De manière très succincte, selon Aoki [2001, p. 29], les institutions ont une nature duale, à la fois contraignante et permissive. C'est également en ces termes que l'on peut donc interpréter les résultats précédents. Plus précisément, dans notre cas, la complémentarité s'opère entre deux domaines institutionnels particuliers : organisationnel et politico-législatif. Par contrainte ou possibilité, un certain environnement juridique conduit à la sélection d'une forme de contrôle particulière, ou inversement.

Deux résultats sont à mettre en avant. En premier lieu, la construction d'indicateurs juridiques du pouvoir actionnarial et leur comparaison statique avec les indicateurs classiques de la protection des actionnaires minoritaires dans 15 pays européens relativisent l'une des conclusions principales de la théorie de l'origine légale. La régulation des conflits d'agence n'apparaît pas comme une condition suffisante à la dispersion de l'actionnariat dès lors que sont pris en compte d'autres éléments juridiques et économiques (cf. tableau 1). Elle ne permet d'ailleurs pas à elle seule d'expliquer la gradation de la concentration de la propriété (cf. tableau 3). Autrement dit, c'est aussi parce que le droit permet aux actionnaires de se retirer du processus décisionnaire en assemblée générale que l’occurrence de structures de propriété dispersées est plus fréquente. L’autonomie des administrateurs, assurée par une

\footnotetext{
${ }^{23}$ Dans le tableau 3C, la statistique $F$ la plus faible que produit la comparaison deux à deux des trois classes est de 3,85. Or, comme l'indique une table de Fisher, cette valeur dépasse le seuil critique de 3,10 de la distribution $F_{1,14}$ avec une marge d'erreur à $10 \%$. L'hypothèse nulle d'égalité des coefficients peut donc être rejetée pour ce cas-là comme pour les deux autres cas du tableau 3C.

${ }^{24}$ Ces auteurs positionnent leur approche en opposition au « canal politique » de Beck et al. [2003] selon lequel les pays de common law assurent une plus grande défense de la propriété privée grâce à une plus grande indépendance du pouvoir judiciaire vis-à-vis du pouvoir législatif.
} 
régulation qui autorise l'éloignement des actionnaires de la gouvernance de la société, est donc une caractéristique organisationnelle préalable à la dispersion de l'actionnariat.

Ce résultat renforce l'une des conclusions à laquelle aboutissent Lele et Siems [2007, p. 35-37] à la suite d'une analyse longitudinale dans cinq pays sur la base d'indicateurs juridiques davantage exhaustifs : il n'y a pas de raison à ce que la transposition dans un pays donné d'un droit favorable à la protection des actionnaires minoritaires se traduise par une modification des modes de gouvernance des firmes. Plus largement, tout cela vient en appui des travaux qui appellent à la prise en compte d'autres éléments juridiques et politiques que les indicateurs classiques de la Law and Finance pour expliquer la gouvernance des firmes (Gourevitch et Shinn [2005] ; Roe [2003, 2006] ; Roe et Siegel [2009]).

Le second résultat concerne quant à lui les trois groupes de pays que met en évidence la cohérence institutionnelle entre les modes de gouvernance et l'environnement juridique des sociétés cotées. En tenant compte de la régulation propre à la diversité des actionnaires, ces différentes classes permettent de dépasser la vision dichotomique entre, d'une part, un modèle de pure autonomie managériale sous dispersion de l'actionnariat et, d'autre part, un modèle de forte concentration de la propriété. L'existence d'une troisième configuration intermédiaire permet notamment de penser les situations de concentration actionnariale dans lesquelles des actionnaires minoritaires sont relativement bien protégés et sont en mesure de jouer un rôle de surveillance des titulaires du contrôle.

Présentons maintenant ces trois configurations. Rappelons que les résultats sont obtenus pour une date postérieure à un processus de convergence formelle et fonctionnelle du droit des actionnaires (Lele et Siems [2007] ; Siems [2008] ; Armour et al. [2009a, b] ; Deakin et Rebérioux [2009] ; Martynova et Renneboog [2011]). Il est donc possible d'estimer qu'ils traduisent la persistance d'une certaine diversité malgré ce processus de convergence à l'œuvre des années 1990 à 2005. Il apparaît au final, d'une part, que chaque juridiction a développé les conditions de préservation de la rente actionnariale des porteurs de titres les plus caractéristiques des principales sociétés cotées ; d'autre part, chacune a facilité les modalités de la prise de décision dans ces sociétés en fonction du niveau de concentration de leur actionnariat.

\section{Le contrôle actionnarial}

Les pays marqués par une forte concentration actionnariale ont conservé ou développé des droits de contrôle et de structure visant la préservation du pouvoir de décision associé à la détention du capital. Les principaux actionnaires des sociétés cotées sont donc en mesure de contrôler un ensemble de décisions affectant le passif ou l'actif de la société (cf. tableau A.1.3., annexe A1). De la même manière, la régulation leur donne davantage de poids dans le processus de sélection des administrateurs - qui à leur tour nomment et surveillent les dirigeants en charge de la gestion quotidienne de la firme (cf. tableau A.1.2., annexe A1). Dans cette configuration du contrôle actionnarial, les administrateurs s'identifient totalement aux actionnaires. Cette relation de contrôle occulte donc complètement les conflits d'agence entre propriétaires et dirigeants.

Les conditions juridiques qui facilitent la prise de décision en assemblée générale permettent alors au groupe de contrôle de nature actionnariale de maîtriser les conditions de production et de préservation de sa rente. Les droits de vote - et les droits de contrôle qui leur sont associés - sont un moyen pour les actionnaires, en nommant les dirigeants qui leur conviennent, d'orienter la gestion de la société vers la maximisation de la valeur actionnariale. 
La défense des intérêts financiers concentrés sur les plus grandes capitalisations boursières ne nécessite donc pas le développement d'une protection des actionnaires minoritaires.

Ici, le canal politique par lequel s’opère le choix de la régulation est direct et correspond à celui mis en avant par Roe (2003) ou Gourevitch et Shinn (2005). Les ressources financières des grands actionnaires, ainsi que leur influence sur une grande part de l'emploi et de l'activité économique domestique, leur confèrent un poids politique important, en suscitant notamment une écoute attentive du législateur. Ces actionnaires de contrôle peuvent alors faire valoir davantage leurs préférences en matière de droit des sociétés que les autres porteurs de titres.

Pour finir, en reprenant les termes d'Aoki et en donnant un autre sens à la causalité entre régulation et gouvernance, il est possible d'énoncer que la sélection d'un mode de contrôle actionnarial est contrainte par une régulation qui impose un vote en assemblée générale sur la plupart des décisions affectant les sociétés de capitaux. Elle l'est d'autant plus que des actionnaires externes relativement petits ne peuvent surveiller le processus décisionnaire en AG. Ils ne sont donc pas incités à prendre des participations trop élevées qui leur permettraient de supporter le coût d'une éventuelle surveillance qui ne serait de toute façon pas soutenue par des dispositifs légaux. De même, les actionnaires internes ne sont pas incités à vendre leurs parts sur le marché au risque de se retrouver à leur tour dans la position des minoritaires, selon l'argument traditionnel de l'expropriation de la Law and Finance. Dans cette situation, la sélection d'un mode de contrôle actionnarial apparaît comme le seul complément institutionnel de cette combinaison particulière associant une faible régulation des conflits d’agence à une bonne qualité juridique du pouvoir actionnarial.

\section{La surveillance actionnariale}

Dans le cas du Royaume-Uni et de l'Irlande, la régulation n'est plus contraignante mais permissive. Elle autorise la sélection d'un contrôle de nature administratoriale mais elle n’empêche pas pour autant les grands actionnaires de prendre le pouvoir en assemblée générale. Un actionnaire de contrôle maîtrisant la composition du conseil d'administration n'a pas en effet à se soucier d'une régulation qui ne soumet pas les décisions de structure à une ratification en assemblée générale puisqu'il contrôle les membres du conseil qui sont en mesure de prendre ces décisions. Cette régulation facilite néanmoins l'exercice d'un contrôle par les administrateurs en limitant le nombre de décisions qui doivent faire l'objet d'un vote en AG. Le Royaume-Uni est le pays typique en la matière : six des onze décisions de structure répertoriées dans le tableau A.1.3. (annexe A1) ne requièrent pas de votes en assemblée générale ; l'indicateur des droits de contrôle du tableau A.1.2. (annexe A1) indique, pour sa part, que le processus de nomination et de révocation des administrateurs des sociétés de ce pays confère à ces derniers une protection et un pouvoir discrétionnaire plus importants que dans d'autres juridictions. La sélection d'un mode de contrôle administratorial et sa viabilité sont par ailleurs assurées par une forte protection des actionnaires minoritaires qui leur garantit une capacité de prise de parole en cas de conflits d'agence.

Ainsi, dans les pays caractérisés par un actionnariat dispersé, les décisions affectant la vie des sociétés de capitaux se prennent de manière plus souple en ce qu'elles ne requièrent pas nécessairement l'implication d'actionnaires davantage concernés par la gestion de leur portefeuille. La régulation du pouvoir actionnarial facilite en ce sens les conditions d'expression et de prise de décisions du groupe de contrôle de nature administratoriale. Mais, en contrepartie, la discrétion plus large des administrateurs est alors soumise à une facilité 
d'intervention des actionnaires minoritaires en assemblée générale. Ceux-ci peuvent donc contester l'administration de la société lorsqu'elle n'est pas gérée dans le sens de la maximisation de la valeur actionnariale et ce, plus facilement que les actionnaires minoritaires d'autres juridictions. La résolution des conflits d'agence est ainsi au cœur de cette régulation.

Ce modèle est cohérent avec la relation que mettent en évidence Kim et al. [2007] entre l'indépendance des conseils d'administration et les indicateurs de LLSV [1998]. Sur un panel de pays européens relativement similaire à celui de cet article, ces auteurs montrent que les pays marqués par la plus forte protection des actionnaires minoritaires sont aussi ceux dont les conseils d'administration comportent le moins de membres issus du management. Plus précisément, les sociétés cotées de l'Irlande et du Royaume-Uni ont des conseils composés d'une majorité de membres indépendants ; ce sont également dans ces pays que les indicateurs de protection des investisseurs sont les plus importants (Kim et al. [2007] p. 867). Ainsi, la gouvernance des firmes appartenant à ces pays présente une certaine autonomie tant à l'égard du management que des actionnaires lorsqu'ils demeurent passifs. Le pouvoir juridique de ces derniers impose néanmoins aux administrateurs de rendre compatibles les stratégies menées par les managers avec les résultats attendus par les investisseurs externes.

\section{Des actionnaires de contrôle sous surveillance actionnariale}

La troisième configuration intermédiaire peut être considérée comme une hybridation des deux précédents modèles, et peut-être moins comme une transition de l'un à l'autre. Autrement dit, la régulation des conflits d'agence peut opérer comme une force de convergence mais n'altère pas les dispositifs associés au contrôle actionnarial ${ }^{25}$. Les principales sociétés des pays de ce modèle sont marquées par une concentration de la propriété tournant autour de $30 \%$, ce qui est un chiffre courant dans les études sur l'actionnariat en Europe (Barca et Becht [2001]). Cela traduit, par rapport aux autres configurations, une occurrence plus fréquente de sociétés sous contrôle actionnarial minoritaire. Les indicateurs du pouvoir actionnarial suggèrent en effet que la maîtrise des décisions en assemblée générale requiert une implication relativement importante des actionnaires. Ainsi, le tableau A.1.3. (annexe A1) montre que la Belgique et le Danemark, qui appartiennent à cette classe, soumettent chacune des onze décisions composant l'indicateur des droits de structure à l'obtention d'une super-majorité en assemblée générale extraordinaire. D'après l'indicateur des droits de contrôle (cf. tableau A.1.2., annexe A1), l'implication en assemblée générale ordinaire est toutefois inférieure à celle qu'impose le modèle du contrôle actionnarial quant à lui davantage marqué par des structures de contrôle majoritaire.

Les administrateurs sont donc désignés par des détenteurs de grands blocs de titres et peuvent être considérés comme proches de ceux qui ont le pouvoir de les nommer et de les révoquer. S’ils administrent la société dans le sens des intérêts des actionnaires de référence, ils doivent néanmoins répondre aux exigences des actionnaires externes en matière de rentabilité ou d'orientation stratégique. Les niveaux de concentration de la propriété et les indicateurs de la protection des minoritaires (cf. figure 1) reflètent en effet la présence active de ces investisseurs. Ces derniers ont ainsi les moyens légaux d'exercer leur fonction de contestation des titulaires du contrôle en cas de désaccord majeur sur la conduite de la firme.

\footnotetext{
${ }^{25}$ Il est ainsi possible d'interpréter cette configuration comme le pendant juridico-économique de l'hypothèse de double convergence que mettent en avant Plihon et al. [2001] au sujet des objectifs propres à chacun des modèles de gouvernance anglo-saxon et européen continental.
} 
Ce pouvoir est néanmoins inférieur à celui dont disposent les investisseurs dans le modèle de la surveillance. Toutefois, si les objectifs privilégiés par les deux types d'actionnaires peuvent diverger, ils demeurent néanmoins chacun guidés par l'objectif de la réalisation d'une valeur actionnariale. Au delà des différences de vue sur les horizons temporels et les niveaux de risque et de rentabilité, les actionnaires de contrôle peuvent donc s'accommoder des préférences des actionnaires surveillants et inversement. Cette configuration incite à une convergence des objectifs entre les deux types de propriétaires de titres sans pour autant conduire à une pure assimilation de leurs préférences.

La logique politique qui sous-tend cette configuration est conforme à l'argumentaire développé par Gourevitch et Shinn [2005] à propos de ce qu'ils nomment le « modèle investisseurs ». Les conclusions apportées ici divergent cependant de celles auxquelles aboutissent ces auteurs. Selon eux, ce modèle est en effet réducteur en ce qu'il postule une convergence d'objectifs entre les différents types d'actionnaires ; il aurait par ailleurs un pouvoir explicatif limité en ce qu'il devrait nécessairement se traduire par une dispersion de l'actionnariat, ce que contredisent les faits stylisés. Le modèle du contrôle actionnarial sous surveillance, au contraire, réconcilie l'hypothèse de la convergence des intérêts avec la persistance d'une certaine concentration de la propriété. L'argument de Gourevitch et Shinn est le suivant : le développement d'une régulation favorable aux actionnaires minoritaires est d'abord le fait des actionnaires de contrôle qui cherchent une amélioration de leurs conditions de financement ou qui souhaitent vendre leurs parts sur le marché. Leur demande politique porte donc sur le développement d'une bonne régulation des conflits d'agence pour répondre aux exigences des investisseurs. L'adoption d'une meilleure protection des minoritaires constitue une réelle amélioration ou ne fait qu'entériner des pratiques déjà fixées dans les statuts des sociétés, par exemple sur la divulgation de l'information ou la limitation de cumul des mandats des administrateurs. Cette demande nationale est par ailleurs soutenue par un ensemble de pressions internationales émanant, par exemple, de la réglementation européenne ou des institutions que sont l'OCDE et la Banque Mondiale.

Cette idée selon laquelle les intérêts des différents types d'actionnaires seraient totalement similaires apparaît excessive. Toutefois, plusieurs propositions en matière de gouvernance d'entreprise émanent des sociétés elles-mêmes et correspondent aux desiderata des investisseurs. Elles se traduisent donc effectivement par des réformes du droit des sociétés dans les pays d'Europe continentale à partir des années 1990 (Enriques et Volpin [2007] ; Plihon et Ponssard [2002]). C'est d'ailleurs ce que reflètent les analyses longitudinales du droit des actionnaires (Lele et Siems [2007] ; Siems [2008]; Martynova et Renneboog [2011]). Ces évolutions concordent avec la thèse avancée par Rajan et Zingales [2003] selon qui les préférences des titulaires du contrôle peuvent être modifiées par la globalisation des marchés de capitaux et par les évolutions sur les marchés des produits - marqués par une concurrence internationale plus intense.

Néanmoins, l'acceptation des actionnaires de contrôle de concéder un pouvoir plus important aux investisseurs externes ne se traduit pas par une transformation radicale des modes de gouvernance. Si un niveau intermédiaire des indicateurs de protection des actionnaires minoritaires peut refléter une certaine convergence vers des standards internationaux, pour ne pas dire anglo-saxons, le niveau équivalent des indicateurs du pouvoir actionnarial traduit au moins le fait que les actionnaires ne sont pas enclins à déserter les structures du pouvoir au sein de la firme. Ce sont précisément les pays de ce modèle du contrôle actionnarial sous surveillance qui incitent à conclure que la protection des minoritaires n'implique pas une dispersion de l'actionnariat. Il existe des déterminants juridiques et organisationnels qui encastrent le choix des actionnaires dans le conseil d'administration. 


\section{CONCLUSION}

Alors que les indicateurs classiques de la Law and Finance avaient pour visée de construire une mesure de la capacité des actionnaires à déterminer la composition d'un conseil d'administration, ceux-ci se sont focalisés sur la protection des actionnaires minoritaires. Cet article propose des indicateurs juridiques du pouvoir actionnarial visant à combler cette lacune. Ces nouvelles mesures sur le poids relatif des administrateurs et des actionnaires portent d'une part sur le contrôle des sociétés cotées, c'est-à-dire sur la capacité d'en nommer et révoquer les administrateurs, et, d'autre part, sur la maîtrise d'un certain nombre de décisions affectant la structure des sociétés de capitaux.

Les indicateurs juridiques du pouvoir des actionnaires construits ici, ainsi que la régulation des conflits d'agence, telle qu'elle est capturée par les indicateurs traditionnels de la Law and Finance, sont alors confrontés pour expliquer la concentration de la propriété des actions. Les deux types de mesure sont complémentaires dans l'explication de la diversité de la concentration actionnariale de 287 sociétés cotées appartenant à 15 pays européens en 2006, une fois contrôlées les caractéristiques individuelles des firmes.

Les juridictions dans lesquelles le droit des actionnaires minoritaires est très développé présentent certes de faibles niveaux de concentration actionnariale mais aussi un plus fort soutien juridique au pouvoir discrétionnaire des administrateurs. Inversement, les pays dans lesquels le pouvoir légal de décision des actionnaires de contrôle est puissant sont marqués par de forts niveaux de concentration actionnariale, et ce en complément de la faiblesse des indicateurs de la protection des minoritaires que la Law and Finance associe généralement à ces pays. Enfin, un certain nombre de juridictions combine, pour les deux types de régulation, des indicateurs de rang intermédiaire, ce qui s'accompagne d'une concentration de l'actionnariat d'un niveau tel qu'elle permet la présence d'actionnaires de contrôle tout en autorisant la surveillance de ces titulaires par la venue et l'expression d'actionnaires minoritaires puissants.

La complémentarité entre les institutions juridiques et économiques ainsi mise en évidence permet d'expliquer pourquoi une protection des actionnaires minoritaires relativement élevée ne se traduit pas nécessairement par une dispersion de l'actionnariat. La régulation du contrôle montre en effet qu'il existe des forces de résistance à un mouvement de morcellement complet de la propriété. Le pouvoir légal des actionnaires de contrôle milite en effet, non pas nécessairement contre le développement de la protection des actionnaires minoritaires, mais pour le maintien d'un statut juridique confortant leur propre position. 


\section{REFERENCES BIBLIOGRAPHIQUES}

Aglietta M. et Reberioux A. [2004], Dérives du capitalisme financier, Paris, Albin Michel, $394 \mathrm{p}$.

AMABLE B. [2005], Les cinq capitalismes : diversité des systèmes économiques et sociaux dans la mondialisation, Paris, Éditions du Seuil, 373 p.

AmABLE B. et Lung Y. [2008], «The European Socio-Economic Models of a Knowledge-based society : main findings and conclusion », Actes du GERPISA, (41), p. 9-23.

AOKI M. [2001], Fondements d'une analyse institutionnelle comparée, Paris, Albin Michel, 604 p., 2006 pour la traduction française.

ARMOUR J., DeAKIN S., LELE P.P., Siems M.M. et Singh A. [2009a], « How legal norms evolve: evidence from a cross-country comparison of shareholder, creditor and worker protection », American Journal of Comparative Law, 57(3), p. 579-629.

ARMour J., DEAKIN S., MolLICA V. et SIEMS M.M. [2010], « Law and Financial Development : What We are Learning from Time-Series Evidence », document de travail n 1580120, SSRN.

ARMour J., DeAKIN S., SARKAR P., Siems M. et SingH A. [2009b], "Shareholder Protection and Stock Market Development: An Empirical Test of the Legal Origins Hypothesis », Journal of Empirical Legal Studies, 6(2), p. 343-380.

AuVRAY T. et GRANIER C. [2009], « La crise financière en Europe : vers une convergence des modèles de détention actionnariale ? », dans DupuY C. et LAVIGNE S. (dir.), Géographies de la finance mondialisée, chap. 5, Paris, La Documentation Française, p. 79-95.

BARCA F. et BECHT M. (dir.) [2001], The Control of Corporate Europe, New York, Oxford University Press, 331 p.

BebchuK L.A., et RoE M.J. [1999], « A Theory of Path Dependence in Corporate Ownership and Governance », Stanford Law Review, 52(1), p. 127-170.

BECHT M., et MAYER C. [2004]. "Corporate Governance in Europe: Competition versus Harmonization », dans Borio C., Hunter W. C., KaufMan G., Tsatsaronis K. (eds), Market Discipline Across Countries and Industries, The MIT Press, p. 255-267.

BECK T., DEMIRGÜÇ-KunT A. et LeVINE R. [2003], « Law and finance : why does legal origin matter ? ", Journal of Comparative Economics, 31(4), p. 653-675.

Belloc M. et PAGANo U. [2009], "Co-evolution of politics and corporate governance », International Review of Law and Economics, 29(2), p. 106 - 114.

Berle A.A., et Means G.C. [1932], The Modern Corporation and Private Property, New York, The MacMillan Company, réédition de 1939.

BerRou J.P. et CARrinCAZEAUX C. [2005], « La diversité des capitalismes et les pays d’Europe Centrale et Orientale : une analyse statistique », Actes du GERPISA, (38), p. 33-82.

Blazy R., Boughanmi A., DefFains B, et Guigou J.-D. [2012], «Corporate governance and financial development: a study of the French case », European Journal of Law and Economics, 33(2), p. 399-445.

CHEVAliER J.-M. [1970], La structure financière de l'industrie américaine, Cujas, Paris, 270 p.

ClaESSENS S., DJANKOV S. et LANG L.H.P. [2000], " The separation of ownership and control in East Asian Corporations », Journal of Financial Economics, 58(1/2), p. 81-112.

DEAKIN S. et REBERIOUX A. [2009], « Les formes actionnariales, les relations salariales et la gestion de l'emploi en Grande-Bretagne et en France : Convergence ou divergence ? ", dans TOUfFut J.P. (dir.), À quoi servent les actionnaires ?, Albin Michel, Paris, p. 65-94.

DEFFAINS B. [2007], "Introduction à l'analyse économique des systèmes juridiques », Revue économique, 58(6), p. 1149-1162.

DJAnKov S., La Porta R., Lopez-De-Silanes F. et Shleifer A. [2003], « Courts », Quarterly Journal of Economics, 118(2), p. 453-517. 
Djankov S., La Porta R., Lopez-De-Silanes F., et Shleifer A. [2008], " The Law and Economics of Self-Dealing », Journal of Financial Economics, 88(3), p. 430-465.

ECGI, ISS et SHEARMAN \& STERLING LLP [2007], Report on the proportionality principle in the European Union, External Study Commissioned by the European Commission.

ENRIQUES L. et VolPIN P. [2007], " Corporate Governance Reforms in Continental Europe », Journal of Economic Perspectives, 21(1), p. 117-140.

Evrard Y., Pras B. et RouX E. [2003], Market : études et recherches en marketing, Dunod, Paris, 699 p., $3^{\mathrm{e}}$ édition.

FACCIO M. et LANG L.H.P. [2002], « The ultimate ownership of Western European corporations », Journal of Financial Economics, 65(3), p. 365 - 395.

FLigSTEIN N. et CHOO J. [2005], « Law and corporate governance », Annual Review of Law and Social Science, 1(1), p. 61-84.

Franks J., MAYER C., et WAGNER H.F. [2006], "The Origins of the German Corporation Finance, Ownership and Control », Review of Finance, 10(4), p. 537-585.

GiBBONS R. [2005], « Four formal(izable) theories of the firm? », Journal of Economic Behavior \& Organization, 58(2), p. $200-245$.

Gourevitch P.A., et ShINN J. [2005] Political Power and Corporate Control:The New Global Politics of Corporate Governance, Princeton University Press, 344 p.

HART O. [1995], Firms, Contracts, and Financial Structure, Clarendon Press, Oxford, 228 p.

HolDERNESS C.G. [2008], " Do Differences in Legal Protections Explain Differences in Ownership Concentration ? », document de travail n 1104678 , SSRN.

HolDERNESS C.G. [2009], " The Myth of Diffuse Ownership in the United States », Review of Financial Studies, 22(4), p. 1377 - 1408.

Hyytinen A., KuOSA I., et TAKAlo T. [2003], « Law or Finance: Evidence from Finland », European Journal of Law and Economics, 16(1), p. 59-89.

KIM K.A., Kitsabunnarat-Chatjuthamard P. et Nofsinger J.R. [2007], « Large shareholders, board independence, and minority shareholder rights : Evidence from Europe », Journal of Corporate Finance, 13(5), p. 859-880.

KIRAT T. [2006], « Les indicateurs de protection de l'emploi : la mesure du droit du travail en question ? », Economie et Institutions, (9), p. 85-127.

LA Porta R., LOPEZ-DE-Silanes F., et Shleifer A. [1999], « Corporate Ownership around the World », The Journal of Finance, 54(2), p. 471-517.

La Porta R., LOPeZ-De-Silanes F., et Shleifer A. [2006], "What Works in Securities Laws? », The Journal of Finance, 61(1), p. 1-32.

La Porta R., LOPEZ-DE-Silanes F. et Shleifer A. [2008], « The Economic Consequences of Legal Origins », Journal of Economic Literature, vol. 46, no 2, p. 285-332.

LA Porta R., LOPEZ-DE-Silanes F., Shleifer A., et VishnY R.W. [1997], « Legal Determinants of External Finance », The Journal of Finance, 52(3), p. 1131-1150.

La Porta R., Lopez-De-Silanes F., Shleifer A., et ViShNY R.W. [1998], "Law and Finance », The Journal of Political Economy, 106(6), p. 1113-1155.

LELE P.P. et SIEMS M.M. [2007], « Shareholder Protection: A Leximetric Approach », Journal of Corporate Law Studies, 7(1), p. 17 - 50.

LLENSE F. [2009], « Rémunérations des P-DG français. Les actionnaires peuvent-ils souhaiter un plafonnement ? », Revue économique, 60(3), p. 759-766.

MARTYNOVA M. et RenNeBOOG L. [2008], «Spillover of corporate governance standards in cross-border mergers and acquisitions », Journal of Corporate Finance, 14(3), p. 200 - 223.

MARTYNOVA M. et RENNEBOOG L. [2011], «Evidence on the international evolution and convergence of corporate governance regulations », Journal of Corporate Finance, 17(5), p. 1531-1557.

MoRIN F. [1974], La structure financière du capitalisme français, Paris, Calmann-Lévy, 316 p. 
MORIN F. [1994], « Liaisons financières et coopération des acteurs-systèmes ", Revue économique, 45(6), p.1459-1470.

PaGAno M., et VolPIN P.F. [2005], «The Political Economy of Corporate Governance », The American Economic Review, 95(4), p. 1005-1030.

PISTOR K., RAISER M. et GELFER S. [2000], «Law and Finance in Transition Economies », Economics of Transition, 8(2), p. 325-368.

PliHON D. et PONSSARD J.P. (dir.) [2002], La montée en puissance des fonds d'investissement : quels enjeux pour les entreprises ?, La documentation française, Paris, 183 p.

PliHON D., PONSSARD J.P. et ZARLOWSKI P. [2001], " Quel scénario pour le gouvernement d'entreprise ? Une hypothèse de double convergence », Revue d'Économie Financière, (63), p. 35-51.

PETIT P. [2006], « D’un capitalisme managérial... à l’autre », Innovations, (23), p. 49-69.

RAJAN R.G. et ZiNGALES L. [2003], « The great reversals : the politics of financial development in the twentieth century ", Journal of Financial Economics, 69(1), p. 5-50.

RoE M.J. [2003], Political Determinants of Corporate Governance: Political Context, Corporate Impact, New York, Oxford University Press, 231 p.

RoE M.J. [2006], « Legal Origins, Politics, and Modern Stock Markets », Harvard Law Review, $120(2)$, p. $462-527$.

RoE M.J. et SiEgel J.I. [2009], "Finance and Politics : A Review Essay Based on Kenneth Dam's Analysis of Legal Traditions in The Law-Growth Nexus ", Journal of Economic Literature, 47(3), p. 781-800.

SHEARMAN \& STERLING LLP [2007], Proportionality between ownership and control in EU listed companies:Comparative legal study, étude externe pour la Commission Européenne.

SIEMS M.M. [2008], "Shareholder Protection around the World [Leximetric II] », Delaware Journal of Corporate Law, 33(1), p. 111 - 147.

SPAMANN H. [2006], « On the Insignificance and/or Endogeneity of La Porta et al.'s "AntiDirector Rights Index" Under Consistent Coding ", Harvard Olin Fellows Discussion Paper $\mathrm{n}^{\circ} 7 / 2006$, Harvard University.

SpamanN H. [2010], " The "Antidirector Rights Index" Revisited », Review of Financial Studies, 23(2), p. 467-486.

TunC A. [1994], «Le gouvernement des sociétés anonymes. Le mouvement de réforme aux États-Unis et au Royaume-Uni », Revue internationale de droit comparé, 46(1), p. 59-72.

VILANOVA L. [2007], "Droit et gouvernance des entreprises. Mythe ou réalité », Revue économique, 58(6), p. 1181-1206. 


\section{ANNEXE A1 : COMPOSITION DES INDICATEURS ET DES VARIABLES}

Tableau A.1.1. Description des indicateurs et des variables

\begin{tabular}{ll}
\hline Variables & Description \\
\hline (1) Variable dépendante (sources : Thomson Reuters - Worldscope ; Amadeus, Bankscope ; rapports annuels) \\
\% concentration & Pourcentage des actions non flottantes (détenues par le premier ou les deux premiers \\
actionnariale & actionnaires stratégiques) en 2006, ou à défaut en 2005 (huit cas).
\end{tabular}

(2) Variables indépendantes (d'après Shearman \& Sterling LLP [2007], codage de l'auteur)

(2.1a) Nomination

Majorité nomination Variable allant de 0 à 1 . On ajoute $1 / 2$ pour chacune des règles suivantes : (1) la majorité nécessaire pour nommer les administrateurs est améliorée (les actions des actionnaires présents ou représentés à l'AG et qui s'abstiennent ou votent blanc sur une résolution sont comptabilisées comme votant « non ») ; (2) la majorité nécessaire pour nommer les administrateurs est une règle obligatoire. Une majorité simple (plus d'actions votant « oui » que votant «non ») assortie d'une règle par défaut vaut 0 .

Représentation des minoritaires Variable allant de 0 à 1 et égale à 1 si un mécanisme permettant la représentation des actionnaires minoritaires au conseil est obligatoire; vaut $1 / 2$ si la représentation nécessite l'inscription dans les statuts, et 0 autrement.

Nomination Score des droits de nomination: somme et moyenne de (1) majorité nomination ; (2) représentation des minoritaires.

(2.1b) Révocation

Majorité révocation

Variable allant de 0 à 1 . On ajoute $1 \frac{1}{2}$ pour chacune des règles suivantes : (1) la majorité nécessaire pour révoquer les administrateurs est améliorée (les actions des actionnaires présents ou représentés à l'AG et qui s'abstiennent ou votent blanc sur une résolution sont comptabilisées comme votant «non»); (2) la majorité nécessaire pour révoquer les administrateurs est une règle obligatoire. Une majorité simple (plus d'actions votant « oui » que votant «non ») assortie d’une règle par défaut vaut 0 .

Révocation ad nutum Variable sur la procédure de révocation allant de 0 à 1 et valant 0 si la révocation s'effectue sans cause seulement. On ajoute $1 / 2$ pour chacune des propositions suivantes : (1) la révocation est sans motif et sans préavis ; (2) la révocation est sans motif et sans indemnités. Un total de 1 correspond à la révocation ad nutum qui s'effectue sans motif, sans préavis et sans indemnités.

Ordre du jour $\quad$ Variable allant de 0 à 1 et valant 1 si la décision de révocation peut être votée sans avoir été inscrite à l'ordre du jour, et 0 autrement.

Révocation

(2.1c) Contrôle

Quorum

Score des droits de révocation : somme et moyenne de (1) majorité révocation ; (2) révocation ad nutum ; (3) ordre du jour.

Variable allant de 0 à 1 et valant 1 si le quorum nécessaire pour nommer et révoquer les administrateurs lors de l'AG en première convocation est d'au moins $20 \%$ des actions votantes (seuil défini par la médiane).

Droits de contrôle Indicateur des droits de contrôle : score allant de 0 à 6 obtenu par la somme de (1) nomination, (2) révocation et (3) quorum; et score allant de 0 à 1 obtenu par la moyenne arithmétique de quorum et des composantes de nomination et révocation.

(2.2) Décisions de structure requérant un vote actionnarial

Droits de structure Indicateur des décisions de structure soumises à un vote en assemblée générale dans au moins un des pays. Score allant de 0 à 11 ou de 0 à 1 pour la moyenne arithmétique. Pour chacune des 11 décisions suivantes, on attribut un codage nul si aucun vote des actionnaires n'est sollicité ; on ajoute $1 / 2$ si un vote des actionnaires est requis à la majorité simple ; on ajoute un point si un vote des actionnaires est requis à la majorité qualifiée au 2/3 au moins. (a) Cinq décisions portent sur la structure du capital : (1) émission de parts ou d'autres titres actions; (2) émission d'obligation ou d'autres instruments de dettes; (3) réduction du capital actions ; (4) rachat d'actions; (5) exemption des droits de préemption statutaires sur de nouvelles actions émises pour raison de trésorerie. (b) Trois décisions portent sur la structure des actifs : (6) fusions ; (7) vente de tous ou de substantiellement tous les actifs (8) liquidation volontaire. (c) Trois décisions portent sur la structure sociale: (9) modification des statuts ; (10) changement de nationalité de la société ; (11) modification de l’objet social. 


\begin{tabular}{|c|c|}
\hline Variables & Description \\
\hline \multicolumn{2}{|c|}{ (2.3) Pouvoir actionnarial } \\
\hline $\begin{array}{l}\text { Indicateur du pouvoir } \\
\text { actionnarial }\end{array}$ & Moyenne arithmétique des moyennes des Droits de contrôle et des Dro \\
\hline \multicolumn{2}{|c|}{ (2.4) Protection des actionnaires minoritaires (sources : DLLS [2008]) } \\
\hline $\begin{array}{l}\text { Indicateur anti- } \\
\text { administrateur }\end{array}$ & $\begin{array}{l}\text { L'anti-director rights index de LLSV [1998] révisé par DLLS [2008], et valable pour } \\
\text { l'année 2003. Score allant de } 0 \text { à } 6 \text {, ou movenne arithmétique allant de } 0 \text { à } 1 \text {. }\end{array}$ \\
\hline $\begin{array}{l}\text { Indicateur anti- } \\
\text { malversation }\end{array}$ & $\begin{array}{l}\text { L'anti-self-dealing index de DLLS [2008] valable pour l'année 2003. Score allant de } 0 \\
\text { à } 1 .\end{array}$ \\
\hline $\begin{array}{l}\text { Protection des } \\
\text { minoritaires }\end{array}$ & $\begin{array}{l}\text { Moyenne de : (indicateur anti-administrateur/6) et indicateur anti-malversation. Score } \\
\text { allant de } 0 \text { à } 1 .\end{array}$ \\
\hline \multicolumn{2}{|c|}{ (3) Variables de contrôle } \\
\hline \multicolumn{2}{|c|}{ (3.1) Variables de contrôle des firmes (sources : Thomson Reuters - Worldscope ; Datastream) } \\
\hline Ln(Capitalisatior & $\begin{array}{l}\text { Logarithme de la capitalisation des sociétés à la fin de l'a } \\
\text { société est radiée dans le courant de l'année } 2006 \text { (1 observat }\end{array}$ \\
\hline Société & $\begin{array}{l}\text { Variable muette valant } 1 \text { si la société appartient aux secteurs de la banque ou de } \\
\text { l'assurance (codes SIC à deux chiffres de } 60 \text { à } 63 \text { inclus). }\end{array}$ \\
\hline & \\
\hline \multicolumn{2}{|c|}{$\begin{array}{l}\text { (3.2) Variables de contrôle des pays (sources : Djankov et al. [2003] ; FMI, World Economic Outlook Database, } \\
\text { Avril 2009; World Federation of Exchanges; OMX) }\end{array}$} \\
\hline Ln(Temps de & Indicateur de Djankov et al. [2003], datant de circa 2000, sur l'efficacité des tribunaux \\
\hline $\begin{array}{l}\text { recouvrement d'un } \\
\text { chèque en blanc) }\end{array}$ & $\begin{array}{l}\text { à résoudre des conflits commerciaux. La variable correspond au log de la durée en jours } \\
\text { calendaires de la procédure judiciaire du recouvrement d'un chèque sans provisions, du } \\
\text { dépôt de la plainte à l'exécution du jugement. }\end{array}$ \\
\hline & \\
\hline $\begin{array}{l}\text { Ln(Capitalisation de } \\
\text { marché/PIB) }\end{array}$ & 2006. \\
\hline
\end{tabular}


Tableau A.1.2. Indicateurs des droits de nomination, de révocation et de contrôle

Ce tableau décrit pour chaque pays les droits des actionnaires relatifs au processus de nomination et de révocation des administrateurs. La définition de chacun des droits est donnée dans le tableau A.1.1.

\begin{tabular}{|c|c|c|c|c|c|c|c|c|c|c|}
\hline \multirow[b]{2}{*}{ Pays } & \multicolumn{3}{|c|}{ Nomination } & \multicolumn{4}{|c|}{ Révocation } & \multicolumn{3}{|c|}{ Contrôle } \\
\hline & $\begin{array}{c}\text { Majorité } \\
\text { nomination }\end{array}$ & $\begin{array}{l}\text { Représentation } \\
\text { des minoritaires }\end{array}$ & $\begin{array}{l}\text { Nomination } \\
\text { (somme) }\end{array}$ & $\begin{array}{l}\text { Majorité } \\
\text { révocation }\end{array}$ & $\begin{array}{l}\text { Révocation } \\
\text { ad nutum }\end{array}$ & $\begin{array}{l}\text { Ordre du } \\
\text { jour }\end{array}$ & $\begin{array}{l}\text { Révocation } \\
\text { (somme) }\end{array}$ & Quorum & $\begin{array}{l}\text { Droits de contrôle } \\
\quad \text { (somme) }\end{array}$ & $\begin{array}{c}\text { Droits de contrôle } \\
\text { (moyenne) }\end{array}$ \\
\hline Allemagne & 0 & 0 & 0 & 0 & $0.5^{* *}$ & 0 & 0,5 & 1 & 1.5 & 0.25 \\
\hline Belgique & 0 & 0.5 & 0.5 & 0.5 & 0.5 & 0 & 1 & 0 & 1.5 & 0.25 \\
\hline Danemark & 0 & 0.5 & 0.5 & 0 & 1 & 0 & 1 & 0 & 1.5 & 0.25 \\
\hline Espagne & 0.5 & 1 & 1.5 & 0.5 & 1 & 1 & 2,5 & 1 & 5 & 0.83 \\
\hline Finlande & 0 & 0 & 0 & 0 & 1 & 0 & 1 & 0 & 1 & 0.17 \\
\hline France & 1 & 0 & 1 & 1 & 1 & 1 & 3 & 1 & 5 & 0.83 \\
\hline Grèce & 0 & 0 & 0 & 0 & 1 & 0 & 1 & 1 & 2 & 0.33 \\
\hline Hongrie & 0.5 & 0 & 0.5 & 1 & 1 & 0 & 2 & 1 & 3.5 & 0.58 \\
\hline Irlande & 0 & 0.5 & 0.5 & 0 & 0 & 0 & 0 & 0 & 0.5 & 0.08 \\
\hline Italie & 0.5 & 1 & 1.5 & 0.5 & 0.5 & 0 & 1 & 1 & 3.5 & 0.58 \\
\hline Luxembourg & 0 & 0.5 & 0.5 & 0.5 & 1 & 1 & 2,5 & 0 & 3 & 0.50 \\
\hline Pays-Bas & 0 & 0 & 0 & 0 & 1 & 0 & 1 & 0 & 1 & 0.17 \\
\hline Pologne & 0.5 & $1^{*}$ & 1.5 & 0.5 & 1 & 0 & 1,5 & 0 & 3 & 0.50 \\
\hline Royaume-Uni & 0 & 0 & 0 & 0 & 0 & 0 & 0 & 0 & 0 & 0.00 \\
\hline Suède & 0 & 0 & 0 & 0 & 1 & 0 & 1 & 0 & 1 & 0.17 \\
\hline
\end{tabular}

Source : d'après Shearman \&Sterling LLP [2007], codage par l'auteur.

* Pour la Pologne, la représentation des actionnaires minoritaires est obligatoire quand le groupe d'actionnaires contrôlant la composition du conseil de surveillance détient au moins 20\% des actions. Toutes les firmes polonaises de notre échantillon dépassent ce seuil comme l'indique le tableau A.2.2, annexe A2.

** L'information n'est pas disponible pour l'Allemagne dans l'étude de droit comparé mobilisée ici. On attribue alors à la variable la valeur moyenne que celle-ci peut prendre. Les résultats sont qualitativement similaires lorsque la variable prend la valeur de 0 ou de 1 . En Allemagne, et en tant que salariés, les membres du directoire ne peuvent faire l'objet d'une révocation ad nutum (Becht et Mayer [2004] ; Shearman \& Sterling LLP [2007]), mais aucune indication assez précise n’a pu être trouvée dans la littérature sur une possible révocation ad nutum des membres du conseil de surveillance (Becht et Mayer [2004] ; Franks et al. [2006]). 
Tableau A.1.3. Indicateurs des droits de structure (détail), des droits de contrôle, et du pouvoir actionnarial

Ce tableau décrit pour chaque pays (a) le détail des droits de structure, (b) leur moyenne, (c) la moyenne de l'indicateur des droits de contrôle, et (d) l'indicateur du pouvoir actionnarial (moyenne arithmétique de (b) et (c)). La définition de chacun des droits est donnée dans le tableau A.1.1.

\begin{tabular}{|c|c|c|c|c|c|c|c|c|c|c|c|c|c|c|c|}
\hline \multirow[t]{2}{*}{ Indicateurs } & \multicolumn{5}{|c|}{ Structure du capital } & \multicolumn{3}{|c|}{ Structure des actifs } & \multicolumn{3}{|c|}{ Structure sociale } & \multirow[b]{2}{*}{ 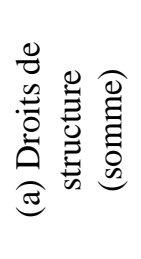 } & \multirow{2}{*}{ 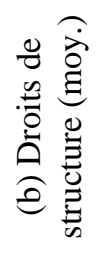 } & \multirow{2}{*}{ 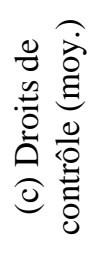 } & \multirow[b]{2}{*}{ 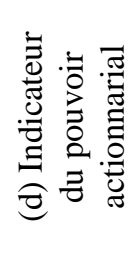 } \\
\hline & 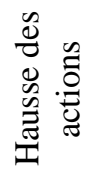 & 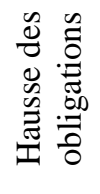 & 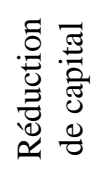 & 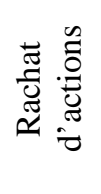 & 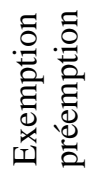 & 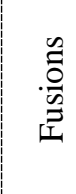 & 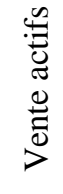 & 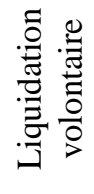 & 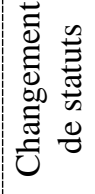 & 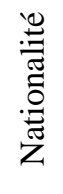 & 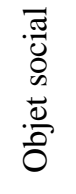 & & & & \\
\hline Allemagne & 1 & 1 & 1 & 0.5 & 1 & 1 & 1 & 1 & 1 & 0 & 1 & 9.5 & 0.86 & 0.25 & 0.56 \\
\hline Belgique & 1 & 1 & 1 & 1 & 1 & 1 & 1 & 1 & 1 & 1 & 1 & 11 & 1.00 & 0.25 & 0.63 \\
\hline Danemark & 1 & 1 & 1 & 1 & 1 & 1 & 1 & 1 & 1 & 1 & 1 & 11 & 1.00 & 0.25 & 0.63 \\
\hline Espagne & 1 & 1 & 1 & 0 & 1 & 1 & 0 & 1 & 1 & 1 & 1 & 9 & 0.82 & 0.83 & 0.83 \\
\hline Finlande & 1 & 0 & 1 & 1 & 1 & 1 & 0 & 1 & 1 & 1 & 1 & 9 & 0.82 & 0.17 & 0.49 \\
\hline France & 1 & 0 & 1 & 1 & 1 & 1 & 0 & 1 & 1 & 0.5 & 1 & 8.5 & 0.77 & 0.83 & 0.80 \\
\hline Grèce & 1 & 1 & 1 & 1 & 1 & 1 & 0 & 1 & 1 & 1 & 1 & 10 & 0.91 & 0.33 & 0.62 \\
\hline Hongrie & 0.5 & 0.5 & 1 & 0.5 & 0.5 & 1 & 0.5 & 1 & 1 & 0.5 & 0.5 & 7.5 & 0.68 & 0.58 & 0.63 \\
\hline Irlande & 1 & 0 & 1 & 1 & 1 & 1 & 0 & 1 & 1 & 0 & 1 & 8 & 0.73 & 0.08 & 0.41 \\
\hline Italie & 1 & 1 & 1 & 0.5 & 1 & 1 & 0 & 1 & 1 & 1 & 1 & 9.5 & 0.86 & 0.58 & 0.72 \\
\hline Luxembourg & 1 & 0 & 1 & 0 & 1 & 1 & 1 & 1 & 1 & 0.5 & 1 & 8.5 & 0.77 & 0.50 & 0.64 \\
\hline Pays-Bas & 0.5 & 0 & 1 & 0 & 1 & 1 & 0 & 0.5 & 0.5 & 0.5 & 0.5 & 5.5 & 0.50 & 0.17 & 0.33 \\
\hline Pologne & 1 & 1 & 1 & 0 & 1 & 1 & 1 & 1 & 1 & 1 & 1 & 10 & 0.91 & 0.50 & 0.70 \\
\hline Royaume-Uni & 0.5 & 0 & 0 & 0 & 1 & 1 & 0 & 0 & 1 & 0 & 1 & 4.5 & 0.41 & 0.00 & 0.20 \\
\hline Suède & 0 & 0 & 1 & 1 & 1 & 1 & 1 & 0 & 1 & 0 & 1 & 7 & 0.64 & 0.17 & 0.40 \\
\hline$\sum$ des décisions requérant une super majorité & 11 & 7 & 14 & 7 & 14 & 15 & 6 & 12 & 14 & 7 & 13 & 120 & $73 \%$ & & \\
\hline$\sum$ des décisions requérant une majorité simple & 3 & 1 & 0 & 3 & 1 & 0 & 1 & 1 & 1 & 4 & 2 & 17 & $10 \%$ & & \\
\hline$\sum$ des décisions ne requérant pas de vote en $\mathrm{AG}$ & 1 & 7 & 1 & 5 & 0 & 0 & 8 & 2 & 0 & 4 & 0 & 28 & $17 \%$ & & \\
\hline
\end{tabular}

Source : d'après Shearman \&Sterling LLP [2007], codage par l'auteur. 


\section{ANNEXE A2. STATISTIQUES DESCRIPTIVES}

Tableau A.2.1. Capitalisation expliquée (en millions d'euros et en \% de la capitalisation boursière du pays)

\begin{tabular}{lrrrrrr}
\hline Pays & Observations & Moyenne & Écart-type & Minimum & Maximum & \% expliqué \\
\hline Hongrie & 19 & 1618.33 & 2865.98 & 52.06 & 9671.96 & $97 \%$ \\
Luxembourg & 14 & 2102.65 & 3808.30 & 133.22 & 12827.17 & $49 \%$ \\
Pologne & 20 & 4012.03 & 3259.56 & 524.04 & 12274.03 & $71 \%$ \\
Irlande & 19 & 5202.93 & 5823.26 & 640.79 & 19702.29 & $80 \%$ \\
Grèce & 18 & 5804.51 & 4202.73 & 1550.00 & 16556.72 & $66 \%$ \\
Danemark & 18 & 7084.14 & 8103.06 & 2021.40 & 29365.04 & $70 \%$ \\
Finlande & 20 & 8304.78 & 13180.90 & 1798.70 & 61389.51 & $71 \%$ \\
Belgique & 20 & 12073.49 & 12332.24 & 1983.66 & 41761.22 & $80 \%$ \\
Suède & 20 & 15131.12 & 11439.85 & 4234.99 & 48664.38 & $64 \%$ \\
Pays-Bas & 19 & 20666.05 & 18897.51 & 6081.56 & 72261.93 & $66 \%$ \\
Espagne & 20 & 24902.38 & 24258.48 & 4206.50 & 88330.00 & $50 \%$ \\
Italie & 20 & 25901.04 & 23847.31 & 7172.13 & 93776.57 & $67 \%$ \\
Allemagne & 20 & 35764.30 & 19718.20 & 7699.80 & 68129.80 & $58 \%$ \\
France & 20 & 49372.44 & 29242.21 & 18571.15 & 123758.60 & $54 \%$ \\
Royaume-Uni & 20 & 74498.96 & 45954.77 & 26979.64 & 167344.73 & $52 \%$ \\
Total & 287 & 20149.49 & 27576.70 & 52.06 & 167344.73 & $58 \%$ \\
\hline
\end{tabular}

Sources : Thomson Reuters - Worldscope ; World Federation of Exchanges et OMX pour la capitalisation totale des pays.

Tableau A.2.2. Pourcentage de la concentration actionnariale

\begin{tabular}{lrrrrr}
\hline Pays & Observations & Moyenne & Écart-type & Minimum & Maximum \\
\hline Royaume-Uni & 20 & 7.25 & 9.63 & 0.01 & 31.87 \\
Allemagne & 20 & 14.41 & 11.12 & 0.08 & 34.80 \\
Irlande & 19 & 17.84 & 16.93 & 0.10 & 55.00 \\
Suède & 20 & 20.30 & 14.03 & 0.08 & 59.01 \\
Finlande & 20 & 24.32 & 19.77 & 0.03 & 50.82 \\
Pays-Bas & 19 & 26.19 & 21.42 & 0.01 & 58.92 \\
France & 20 & 28.52 & 28.23 & 1.11 & 89.22 \\
Italie & 20 & 30.34 & 23.95 & 0.35 & 86.27 \\
Espagne & 20 & 35.73 & 21.88 & 5.15 & 68.01 \\
Danemark & 18 & 38.78 & 30.78 & 0.03 & 92.60 \\
Grèce & 18 & 42.37 & 21.06 & 6.00 & 71.97 \\
Belgique & 20 & 43.70 & 22.52 & 0.81 & 98.62 \\
Luxembourg & 14 & 56.23 & 26.24 & 15.86 & 91.18 \\
Hongrie & 19 & 57.39 & 25.72 & 11.43 & 96.80 \\
Pologne & 20 & 61.88 & 19.40 & 21.94 & 97.96 \\
Total & 287 & 33.11 & 26.18 & 0.01 & 98.62 \\
\hline
\end{tabular}

Sources : Thomson Reuters - Worldscope, Amadeus, Bankscope et rapports annuels des sociétés. 
Tableau A.2.3. Statistiques descriptives et table de corrélation des principales variables (287 observations)

\begin{tabular}{|c|c|c|c|c|c|c|c|c|c|c|c|c|c|c|c|c|c|}
\hline & Variables & Moy. & E.T. & Min. & Max. & 1 & 2 & 3 & 4 & 5 & 6 & 7 & 8 & 9 & 10 & 11 & 12 \\
\hline 1 & Droits de contrôle & 0.37 & 0.25 & 0 & 0.83 & 1.000 & & & & & & & & & & & \\
\hline 2 & Droits de structure & 0.78 & 0.16 & 0.41 & 1 & 0.320 & 1.000 & & & & & & & & & & \\
\hline 3 & $\begin{array}{l}\text { Indic. du pouvoir } \\
\text { actionnarial }\end{array}$ & 0.57 & 0.17 & 0.20 & 0.83 & 0.892 & 0.713 & 1.000 & & & & & & & & & \\
\hline 4 & Droits anti-administrateur & 0.54 & 0.18 & 0.33 & 0.83 & -0.246 & -0.259 & -0.305 & 1.000 & & & & & & & & \\
\hline 5 & Droits anti-malversation & 0.41 & 0.21 & 0.18 & 0.95 & -0.472 & -0.282 & -0.484 & 0.711 & 1.000 & & & & & & & \\
\hline 6 & $\begin{array}{l}\text { Indic.de la protection des } \\
\text { actionnaires minoritaires }\end{array}$ & 0.48 & 0.18 & 0.26 & 0.89 & -0.396 & -0.293 & -0.432 & 0.915 & 0.934 & 1.000 & & & & & & \\
\hline 7 & $\operatorname{Ln}(\mathrm{PIB} / \mathrm{POP})$ & 10.40 & 0.54 & 9.10 & 11.41 & -0.367 & -0.148 & -0.342 & 0.408 & 0.374 & 0.422 & 1.000 & & & & & \\
\hline 8 & $\begin{array}{l}\text { Ln(temps de recouvrement } \\
\text { d'un chèque en blanc) }\end{array}$ & 5.25 & 0.78 & 3.66 & 6.91 & 0.429 & 0.383 & 0.500 & -0.527 & -0.276 & -0.425 & -0.639 & 1.000 & & & & \\
\hline 9 & Ln(Capitalisation) & 8.97 & 1.60 & 3.95 & 12.03 & -0.051 & -0.211 & -0.139 & 0.384 & 0.287 & 0.359 & 0.223 & -0.280 & 1.000 & & & \\
\hline 10 & Durée de cotation & 19.29 & 10.99 & 2 & 43 & -0.210 & -0.140 & -0.222 & 0.289 & 0.274 & 0.304 & 0.324 & -0.362 & 0.362 & 1.000 & & \\
\hline 11 & Société financière & 0.26 & 0.44 & 0 & 1 & 0.032 & 0.041 & 0.043 & -0.050 & 0.035 & -0.005 & -0.094 & 0.144 & 0.165 & 0.057 & 1.000 & \\
\hline 12 & $\begin{array}{l}\text { Ln(Capitalisation de } \\
\text { marché/PIB) }\end{array}$ & 4.44 & 0.47 & 3.56 & 5.18 & -0.297 & -0.372 & -0.398 & 0.404 & 0.322 & 0.389 & 0.739 & -0.545 & 0.268 & 0.150 & -0.120 & 1.000 \\
\hline
\end{tabular}

